\title{
ASYMPTOTIC PROPERTIES OF DEPTH ONE FOLIATIONS IN HYPERBOLIC 3-MANIFOLDS
}

\author{
SÉRGIO R. FENLEY
}

\begin{abstract}
Let $M$ be a closed hyperbolic 3-manifold with a Reebless depth one foliation $\mathscr{F}$ such that no compact leaf is a fiber of $M$ over $S^{1}$. Assume the monodromies associated to the depth one leaves are irreducible. We show that a compact leaf is associated to a quasi-Fuchsian group, so its limit set is a Jordan curve. For a depth one leaf $T$ embedded with a fixed hyperbolic metric, we show that the embedding of $\widetilde{T}=\mathbf{H}^{2} \rightarrow \mathbf{H}^{3}$ between universal covers extends continuously to the circle at infinity. The image of this circle at infinity has measure 0 and is a Sierpinski curve. The foliation naturally produces a "distance" function which is quasi-isometric to the hyperbolic metric in the universal cover. This is the essential tool used in the study of the limit sets of leaves.
\end{abstract}

\section{Introduction}

Reebless foliations have been extensively used to study the topology of 3-manifolds. For example, Novikov [15] showed that when $M$ is closed the existence of a Reebless, codimension one foliation implies that $\pi_{2}(M)$ is trivial, the fundamental group is infinite, leaves are incompressible surfaces, and transversals are nontrivial in homotopy. These results can be interpreted as a kind of topological rigidity for foliations.

The purpose of this article is to show that under certain conditions the foliation and a geometric structure are also strongly related. One important problem is to determine how efficient the foliation is in measuring distances between points in a fixed homotopy class, which amounts to asking how tight the foliation is.

We restrict to $M^{3}$ connected, closed, hyperbolic. This is a large class, especially important in terms of this question because they share many properties with manifolds that admit Reebless foliations and they exhibit a rich geometric behavior. Since the problem is about homotopy classes we lift to the universal cover, which will then be foliated by topological

Received August 27, 1990 and, in revised form, March 19, 1991. 
planes. We analyze the asymptotic behavior of leaves in the standard compactification of $\mathbf{H}^{3}$ with the sphere at infinity, $S_{\infty}^{2}$. In many cases a given leaf has an induced metric which is quasiconformal to a hyperbolic metric. Therefore one can think of its universal cover as $\mathbf{H}^{2}$, which also can be canonically compactified with a circle at infinity. This situation was analyzed by Cannon and Thurston [2] when the foliation is a fibration over the circle. Their study depends on properties of pseudo-Anosov maps of surfaces because the monodromy of the fibration is of this type. It is an easy fact that in the universal cover the leaves cannot be quasiisometrically embedded, but they show that the inclusion maps $\mathbf{H}^{2} \rightarrow \mathbf{H}^{3}$ extend continuously to the circle at infinity, which have sphere filling curves as images.

In this article we study the case of Reebless, depth one foliations. Intuitively they are fibrations over the circle in the complement of the compact leaves (the compact leaves are the depth 0 leaves). Noncompact leaves have infinite genus surfaces and are the depth one leaves, their only limit points in $M$ are in compact leaves. We can assume no compact leaf is a fiber of $M$ over $S^{1}$, for in that case the analysis reduces to [2]. Our main result is, given a leaf $T$, the embedding of $\widetilde{T}$ into $\mathbf{H}^{3}$ extends continuously to the circle at infinity, as long as some technical conditions on the foliation are satisfied. Here again the extension will not in general be an embedding of the closed disk into the closed 3-ball. For a depth one leaf, the image of the circle at infinity will be the complement of a dense, countable union of open disks, that is, a Sierpinski curve.

The extension result for the compact leaves follows from a general theorem of Thurston, about surfaces in hyperbolic 3-manifolds. On the other hand, the analysis of the depth one leaves depends strongly on the structure of the foliation. The basic idea is to again reduce the study of 3dimensional geometry to 2-dimensional geometry plus an analysis of its homeomorphisms. The monodromy of the fibration in a component $(N)$ of the complement of the compact leaves is an end periodic map: it is a homeomorphism of an infinite genus surface which is topologically trivial everywhere but in a compact subsurface. These maps were studied by Handel and Miller [10] and are similar to maps of finite genus surfaces because of the property above. Up to isotopy of the map there are invariant transverse geodesic laminations and transverse measures.

One difference from the finite genus case is that $M$ hyperbolic does not at all imply the homeomorphism is irreducible [4] and irreducibility is a hypothesis in our work. Furthermore even in the irreducible case it is not always true that the transversal measures have full support [5]. As 
a matter of fact the full support case, also called pseudo-Anosov, is the nongeneric case. To circumvent this we construct projectively invariant transverse measures whose support strictly contains the stable and unstable laminations.

The transversal measures produce a degenerate metric in $\widetilde{N}$ (called a semimetric), which after adjustments in neighborhoods of lifts of compact leaves will be quasi-isometric to the hyperbolic metric. A pseudo-Anosov homeomorphism of a compact surface controls, up to a bounded distortion, the geometry of the universal cover of the circle bundle associated to it. This important consequence of the pseudo-Anosov theory carries over to our case because of the essential finiteness of the end periodic maps and a weak pseudo-Anosov like property in the irreducible case.

This implies that the foliation is only flexible up to a certain extent and it controls the geometry of the universal cover up to a bounded distortion. This is a form of geometrical rigidity associated to the foliation and for many applications in hyperbolic manifolds this property is as good as having no distortion. The theorem about limit sets of foliations can be thought of as its first consequence.

A recurring problem here is that the distance function constructed is not a metric; for example, minimal paths are not unique (even locally) and they may have infinite hyperbolic length. This will introduce some technicalities.

The idea in constructing the extension is to use the foliations and laminations to produce a neighborhood basis of an ideal point in $\mathbf{H}^{3}$ associated to an ideal direction in the leaf. The continuity is proved by $\varepsilon, \delta$ arguments and also depends strongly on properties of the foliation.

We state these results formally. Let $\rho$ be a fixed representation of $\pi_{1}(M)$ into $\mathrm{PSL}_{2}(\mathbf{C})$. Let $T$ be a leaf of the foliation $\mathscr{F}$ and put a hyperbolic structure on $T$ which is quasiconformal to the path metric induced from the inclusion in $M$. Lift $\mathscr{F}$ to a foliation $\widetilde{\mathscr{F}}$ by topological planes of $\widetilde{M}=\mathbf{H}^{3}$. Let $\varphi_{\widetilde{T}}$ be the map from $\mathbf{H}^{2}$ to $\mathbf{H}^{3}$ associated to a lift $\widetilde{T}$ of $T$.

Theorem 1.1. Let $\mathscr{F}$ be a codimension one, Reebless, transversely orientable, depth one foliation on a closed hyperbolic 3-manifold $M$. Suppose that no compact leaf is a fiber of a fibration of $M$ over $S^{1}$. Assume the monodromies associated to the infinite genus leaves are irreducible. Then:

(a) If $T$ is any leaf of $\mathscr{F}, \varphi_{\widetilde{T}}: \mathbf{H}^{2} \rightarrow \mathbf{H}^{3}$ extends continuously to the circle at infinity for any lift $\widetilde{T}$ of $T$ to the universal cover of $M$, so $\left.\varphi_{\widetilde{T}}\right|_{S_{\infty}^{1}}$ gives a parametrization of the limit set of $\tilde{T}$. 
(b) If $S$ is a compact leaf then $\rho\left(\pi_{1}(S)\right)$ is a quasi-Fuchsian group and so has a quasi-circle as a limit set.

(c) If $T$ is a depth one leaf then its limit set is a Sierpinski curve of measure 0 , therefore not a Jordan curve nor the whole sphere.

The paper is organized as follows: in the next section basic definitions are given and the result about the compact leaves is proved. $\S 3$ is a review on end periodic homeomorphisms, where the technical hypothesis for the depth one leaves are explained. In $\S 4$ the problem for the noncompact leaves is reduced to a standard case and the idea of the proof for these leaves is given. The next 2 sections contain the needed technical results: in $\S 5$ we introduce the semimetric in a component of the complement of the compact leaves and show it is quasi-isometric to the hyperbolic metric in the corresponding universal cover. We also extend the semimetric to the whole manifold and again show quasicomparability. In $\S 6$ we show that some naturally defined 2-dimensional submanifolds are quasigeodesic. They will be the key ingredient to produce a neighborhood basis of the desired ideal points. Finally, $\S 7$ we show the extension theorem for the infinite genus leaves.

\section{Definitions and the compact leaf case}

Any closed 3-manifold has a codimension one foliation (see [13], [15]), but these easily constructed foliations are not incompressible. This property which is essential in the theory of foliations means that the fundamental group of a given leaf injects into $\pi_{1}(M)$. The classical theorem of Novikov [15] states that every codimension one foliation of a 3-manifold with a compressible leaf has a Reeb component.

The existence of incompressible foliations was proved constructively by Gabai [6], [7] whenever $H_{2}(M) \neq 0$. These are taut, that is, through every leaf passes a closed transversal, a fact stronger than having no Reeb components. Furthermore some of these foliations are of finite depth, which we describe now. A leaf is proper if it does not limit on itself, and a foliation is totally proper (or more simply, proper) if all leaves are proper. The compact leaves are the depth 0 leaves and, inductively, a leaf is at depth $k$ if all its limit points belong to leaves at depth $<k$ and there are limit points at depth $k-1$. A proper foliation has finite depth $k$ if all leaves have finite depth and $k$ is the maximum of the depths.

We will deal with depth one foliations in 3-manifolds. They are quite common: if $K$ is a knot in $S^{3}$ which either has a presentation with $\leq 10$ crossings or is alternating, then $S^{3}-N(K)$ has a taut foliation transverse 
to the boundary of depth $\leq 1$ (and many of them are not fibrations) (see [7]).

A codimension one foliation is transversally orientable if there is a continuous choice of a transversal vector field. This generates the transversal flow. There is always a covering space of $M$ of order $\leq 4$ which is orientable and where the lifted foliation is transversally orientable. Since we want to study properties in the universal cover we assume from now on that both these properties hold.

A hyperbolic 3-manifold is a Riemannian manifold with constant sectional curvature -1 . Its universal cover is isometric to a subset of $\mathbf{H}^{3}$. References for hyperbolic geometry and hyperbolic 3-manifolds are [1], [14], [17], [18].

In this article limit point is used with two different meanings: (1) in $M$ corresponding to a limit point of a leaf of the foliation and (2) in $\widetilde{M}$ corresponding to an ideal limit point in $S_{\infty}^{2}$ of the lift of a leaf or of any given set. The context will make clear which sense we are referring to.

Definition. A semimetric on $M$ is a function $d: M \times M \rightarrow \mathbf{R}$ satisfying: there exists a $\theta>0$ so that for any $x, y, z, \in M$

(i) $d(x, y) \geq 0, d(x, x)=0$,

(ii) $d(x, y)=d(y, x)$,

(iii) $d(x, y) \leq d(x, z)+d(z, y)+\theta$.

Definition. A quasi-isometry is an injective map $i:(M, d) \rightarrow\left(M^{\prime}, d^{\prime}\right)$ between semimetric spaces such that there are $K, k>1$ satisfying:

$$
\begin{aligned}
& \forall x, y \in M, \max \left(d^{\prime}(i(x), i(y)), d(x, y)\right)>K \\
& \Rightarrow \frac{1}{k} d(x, y) \leq d^{\prime}(i(x), i(y)) \leq k d(x, y) .
\end{aligned}
$$

This is equivalent to finding $k, K>0$ so that, for any $x, y \in M$, $d^{\prime}(i(x), i(y)) \leq k d(x, y)+K$ and $d(x, y) \leq k d^{\prime}(i(x), i(y))+K$. Two semimetrics in a space $M$ are said to be quasi-isometric if the identity map id: $(M, d) \rightarrow\left(M, d^{\prime}\right)$ is a quasi-isometry.

Let $d$ be a Riemannian metric on a space $X$ and $\gamma$ a rectifiable curve in $X$. Then $\gamma$ is an $(r, u)$ quasigeodesic curve $(r, u>0)$ if it satisfies the following property: given a subarc $\gamma^{\prime}$ of $\gamma$ with endpoints $p, q$, if its length is $>u$ then $l\left(\gamma^{\prime}\right) \leq r d(p, q)$. The intuitive idea is that these paths are efficient up to a bounded distortion to measure distance in the metric space. The specification $(r, u)$ will be omitted when not strictly necessary.

If $S$ is an embedded surface in $M$ and $\rho\left(\pi_{1}(S)\right)$ is a quasi-Fuchsian group [17] then once a hyperbolic structure on $S$ is fixed, any given lift 
$\varphi: \widetilde{S}=\mathbf{H}^{2} \rightarrow \mathbf{H}^{3}$ is a quasi-isometry [17]. This implies that geodesics in $\widetilde{S}$ are mapped into $(r, u)$ quasigeodesics in $\mathbf{H}^{3}$ for fixed $(r, u)$ [17, Chapter 8]. It also implies [17, Chapter 11] that the limit set of $\varphi(\widetilde{S})$ is a Jordan curve and the extension of $\varphi$ to $\mathbf{H}^{2} \cup S_{\infty}^{1}$ is continuous.

A quasigeodesic set in a Riemannian manifold $X$ is a set $E \subset X$ satisfying: there is $\mu>0$ so that for any $p, q \in E$ there is a minimal length geodesic connecting them which is at most a distance $\mu$ from $E$. An example is the set $\varphi(\widetilde{S}) \subset \mathbf{H}^{3}$, where $S$ corresponds to a quasi-Fuchsian group. The context will make clear whether we are referring to quasigeodesic curves or sets.

If a leaf $F$ of $\mathscr{F}$ lifts to a fiber of a fibration over $S^{1}$ in a finite cover of $M$, then by classical 3-manifold topology [11], [12], [19] either $M$ fibers over the circle with fiber $F$ or $M$ is the union of two twisted $I$ bundles over $F$ glued along the common boundary $F$. The second possibility does not occur because $\mathscr{F}$ is a transversely orientable foliation.

If $f: S \rightarrow M$ is an immersion, injective on $\pi_{1}$, and $M$ is a closed hyperbolic 3-manifold, then, by [17, Chapters 8,9$]$ and the proof of the hyperbolization theorem for Haken manifolds [14], either $\rho\left(f_{*}\left(\pi_{1}(S)\right)\right)$ is a quasi-Fuchsian subgroup of $\rho\left(\pi_{1}(M)\right)$ or $S$ lifts to a fiber in some finite cover of $M$.

Let $S$ be a compact leaf of $\mathscr{F}$. Since $\mathscr{F}$ is Reebless, Novikov's theorem [15] implies that $S$ is incompressible. Then as $M$ is hyperbolic and closed, $\chi(S)<0$ (by [8] this implies that the foliation is taut also) so $S$ admits a hyperbolic metric. Fix one such metric. The following result is not immediate. Notice it does not require that $M$ does not fiber over $S^{1}$.

Corollary 2.1. Let $\mathscr{F}$ be a codimension one, Reebless, depth 1, transversely orientable foliation in a closed hyperbolic 3-manifold which does not have a compact leaf which is a fiber over $S^{1}$. Then any compact leaf $S$ corresponds to a quasi-Fuchsian subgroup of $\rho\left(\pi_{1}(M)\right)$. In particular, any lift $\varphi: \widetilde{S} \cong \mathbf{H}^{2} \rightarrow \mathbf{H}^{3}$ is a quasidisk, so it extends continuously to a map $\bar{\varphi}: \mathbf{H}^{2} \cup S_{\infty}^{1} \rightarrow \mathbf{H}^{3} \cup S_{\infty}^{2}$ and $\bar{\varphi}\left(S^{1}\right)$ is a Jordan curve in $S_{\infty}^{2}$, which is a quasicircle.

\section{End periodic homeomorphisms}

In this section we describe the main results of the end periodic theory according to [10]. An end periodic surface is a noncompact surface $T$ with finitely many ends so that there is a homeomorphism of $f$ of $T$ satisfying: if $e$ is an end of $T$ there is a neighborhood $U_{e}$ of it so that 


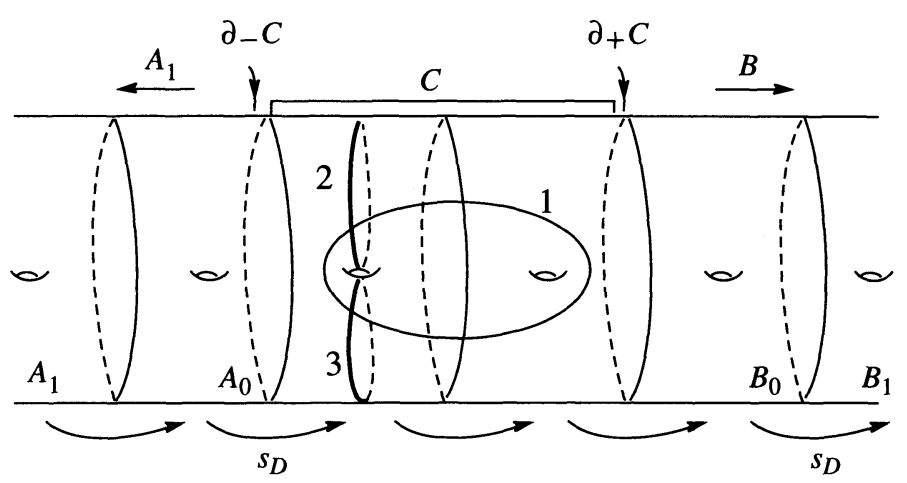

FIGURE 1. AN END PERIODIC SURFACE WITH AN END PERIODIC MAP.

$f\left(U_{e}\right) \subset U_{e}$ (or $U_{e} \subset f\left(U_{e}\right)$ ), a strict inclusion. Furthermore for any neighborhood $U_{e}$, the $f^{n}\left(U_{e}\right), n \geq 0 \quad(n \leq 0)$, form a neighborhood basis of this end which will then be an attracting (repelling) end. The map $f$ is called an end periodic homeomorphism. Figure 1 shows an example of such a map. The surface is the bi-infinite stack of (2-dimensional torus -2 disks) glued consecutively. The ends correspond to $A$ and $B$. The homeomorphism depicted is $f=d_{3} d_{2} d_{1} s_{D}$, where $s_{D}$ is the shift of one block to the right and $d_{i}$ is the Dehn twist around curve $i$.

Give $D \subset T, D \neq T, \partial D$ denotes the relative boundary of $D$ in $T$, namely the closure of the set of boundary points of $D$ which are not boundary points of $T$. In general there is an open neighborhood $U_{e}$ of $e$ so that $\partial U_{e}$ is a separating simple closed curve or disjoint union of properly embedded arcs so that $f\left(\partial U_{e}\right) \subset U_{e}\left(\right.$ or $\left.f^{-1}\left(\partial U_{e}\right) \subset U_{e}\right)$.

If $T$ is topologically hyperbolic, fix one hyperbolic structure on $T$ with geodesic boundary, for which $f$ is an isometry on the ends. In fact it suffices to have one which is quasiconformal to this one, for the only problematic behavior occurs when the metric blows up deep in the ends.

A closed reducing curve is a nonperipheral closed curve $\gamma$ so that for some $n, m \in \mathbf{Z}, f^{n}(\gamma) \subset$ an attracting end and $f^{m}(\gamma) \subset$ a repelling end (obviously $m<n$ ). We call such a curve escaping because its iterates move from $-\infty$ to $+\infty$. After cut and paste operations, such a curve produces a simple closed curve which is disjoint from all its translates and satisfies the property above. One can then cut along the orbit of the curve to obtain a simpler end periodic surface and an induced end periodic 
homeomorphism. This is a reduction. A reducing arc is a nonperipheral, embedded, infinite proper arc, with one end in a repelling end the other in an attracting one, which is periodic up to a proper homotopy (preserving deepness in the ends). A periodic curve is a nonperipheral, closed curve periodic up to homotopy under $f$. The homeomorphism is irreducible if there are no closed reducing curves or reducing arcs or periodic curves and the ends are not simply connected.

Consider now an irreducible homeomorphism $f$ on $T$, an end periodic surface with geodesic boundary. For each end $e$ choose $U_{e}$ open, so that $\partial U_{e}$ is geodesic and $f\left(\partial U_{e}\right) \subset U_{e}\left(f^{-1}\left(U_{e}\right) \subset U_{e}\right)$. Let $U_{+}\left(U_{-}\right)=$ $\cup U_{e}$, where $e$ runs through the attracting (repelling) ends. Let $C=$ $T-\left(U_{-} \cup U_{+}\right)$, which is a finite genus surface called the core of $T$. For simplicity we assume here that $C$ is compact. The set $U_{+}\left(U_{-}\right)$is the positive (negative) ladder. The positive (negative) juncture is $\partial U_{+}\left(\partial U_{-}\right)$. The same notation is used for the lifts to $\widetilde{T}$. Set $\partial_{+} C=\partial U_{+}, \partial_{-} C=$ $\partial U_{-}$. Given a curve $\gamma$ let $\gamma^{*}$ be the geodesic arc homotopic to it and if $\gamma$ is closed let $\gamma^{*}$ be the closed geodesic which is freely homotopic to it.

Theorem 3.1 [10]. Let $f: T \rightarrow T$ be an irreducible end periodic homeomorphism in an end periodic surface with geodesic boundary. Then $f$ is isotopic to $g$, end periodic so that:

(i) $g^{n}\left(\partial_{+} C\right)=\left(f^{n}\left(\partial_{+} C\right)\right)^{*}, \forall n \in \mathbf{Z}$, and similarly for $\partial_{-} C$.

(ii) $g$ preserves transverse geodesic laminations $\Gamma^{u}$ (unstable) and $\Gamma^{s}$ (stable) so that $\Gamma^{u} \subset C \cup U_{+}$and $\Gamma^{s} \subset C \cup U_{-}$.

(iii) $\left(\Gamma^{u} \cup \Gamma^{s}\right) \cap C$ weakly binds $C$ in the sense that complementary regions have compact closure and those which are not peripheral are simply connected.

The laminations are obtained as follows: The set $\partial_{-} C$ is a 1 -manifold disjoint from all its images by $f$. It is called a juncture. Therefore $\gamma_{n}=$ $\left(f^{n}\left(\partial_{-} C\right)\right)^{*}, n \in \mathbf{Z}$, is a finite union of disjoint simple geodesics and arcs. If some $\gamma_{n}$ is contained in $U_{+}$the map is just a shift because $f^{n}\left(\partial_{-} C\right)$ is separating. Otherwise for $n>0$ this set always intersects $C$. Then $\Gamma^{u}$ is the geometric limit of this sequence as $n \rightarrow+\infty$. Let $\mathscr{U}_{+}=\bigcup_{n \in \mathbf{Z}}\left(g^{n}\left(U_{+}\right)\right)$ and similarly define $\mathscr{U}_{-}$. These sets are open and as $\partial_{+} C\left(\partial_{-} C\right)$ are separating curves and $g^{-1}\left(\partial_{-} C\right) \subset U_{-}$it follows that $\Gamma^{u}=\partial \mathscr{U}_{-}$and similarly $\Gamma^{s}=\partial \mathscr{U}_{+}$.

Let $W_{+}=\bigcup_{n \in \mathbf{Z}}\left(g^{n}\left(\partial_{-} C\right)^{*}\right), V_{+}=\Gamma^{u} \cup W_{+}$and similarly define $W_{-}$, $V_{-}$, all geodesic laminations. Then the connected components of $\left(V_{+}-V_{-}\right)$ and $\left(V_{-}-V_{+}\right)$are bounded length intervals. The construction of $g$ is done 


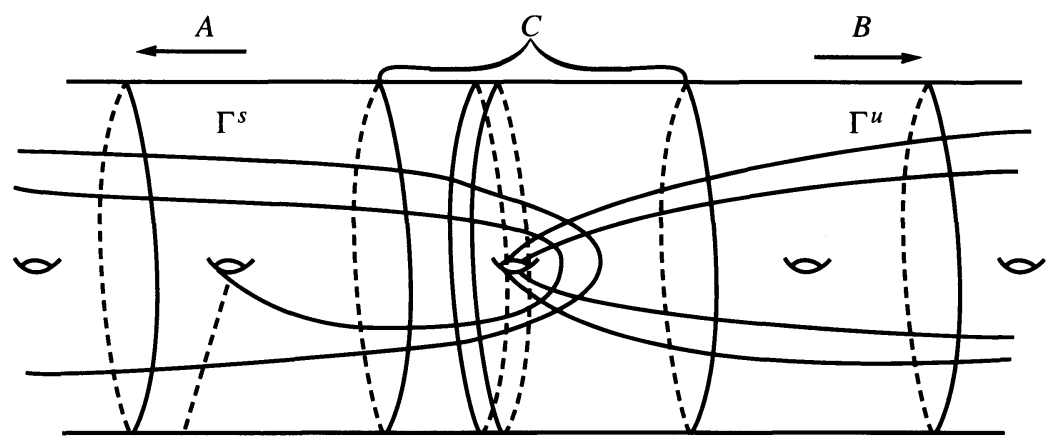

Figure 2. The INVARIANT LAMinations.

so that $\left(g^{n}\left(\partial_{-} C\right)\right)^{*}=g^{n}\left(\partial_{-} C\right)$ which implies $\Gamma^{u}=\lim _{n \rightarrow+\infty} g^{n}\left(\partial_{-} C\right)$ and $V_{+}$is invariant under $g$. Furthermore if $l$ is a component of $\left(V_{+}\right.$ $V_{-}$) then the closure of $l$ is taken to its image by $g$ so that distances are expanded (or contracted) uniformly. Similar statements hold for $V_{-}$.

As the $\left(f^{n}\left(\partial_{-} C\right)\right)^{*}$ are disjoint geodesics and $C$ is compact it follows that $\left(g^{n}\left(\partial_{-} C\right)\right) \cap C, n>0$, is a union of compact arcs which are isotopic rel $\partial_{+} C$ to a finite number of simple, properly embedded arcs of $C$ with boundary in $\partial_{+} C$. Therefore the same holds for $V_{+} \cap C$. Analogous results hold for $V_{-}$. The components of $T-\left(V_{-} \cup V_{+}\right)$have compact closure and are either simply connected or peripheral.

Let $A_{0}=U_{-}-\left(g^{-1}\left(U_{-}\right)\right)$. Since $U_{-}$is a union of neighborhoods of finitely many ends, $A_{0}$ is a finite genus surface. If $A_{i}=g^{-i}\left(A_{0}\right)$ then $A=U_{-}=\bigcup_{i \geq 0} g^{-i}\left(A_{0}\right)=\bigcup_{i \geq 0} A_{i}$ and $A_{i}$ intersects $A_{i+1}$ only in the boundary (which are iterates of junctures). Similarly for the positive ladder $U_{+}=B=\bigcup_{i \geq 0} B_{i}$.

Intuitively one expects that every point $x$ not in either lamination is an escaping point, so that there are $n, m \in \mathbf{Z}$ with $g^{n}(x) \in U_{-}$and $g^{m}(x) \in U_{+}$. It turns out that this is not true in general. A set in $\bar{T}$ is an immersed cusp if it is a hyperbolic geodesic triangle with one ideal vertex. A quadrilateral component of $T-\left(V_{-} \cup V_{+}\right)$is a quadrilateral with two opposite sides in $V_{+}$and the other two in $V_{-}$.

Proposition 3.2 [10]. Consider $D_{1}, \cdots, \bar{D}_{m}$, the components of $T-$ $\left(V_{-} \cup V_{+}\right)$contained in the core, which are not quadrilaterals and are disjoint from $\mathscr{U}_{-}$and $\mathscr{U}_{+}$. Then each $D_{i}$ is a finite sided polygon with alternating sides in $V_{+}, V_{-}$. Furthermore, $T-\left(\Gamma^{u} \cup \mathscr{U}_{-}\right)=\Delta^{+}=\Delta_{1}^{+} \cup \cdots \cup \Delta_{m}^{+}$(disjoint union), where $D_{i} \subset \Delta_{i}^{+}$and $\Delta_{i}^{+}-D_{i}$ is a finite union of immersed cusps. 
Each $\Delta_{i}^{+}$is an ideal geodesic polygon, embedded in $T$. Finally there is an iterate of $g$ which sends $D_{i}$ to itself and pointwise fixes the vertices of $\bar{D}_{i}$ (and therefore fixes $\left.\Delta_{i}^{+}\right)$. Similar statements holds for $T-\left(\Gamma^{\mathcal{S}} \cup \mathscr{U}_{+}\right)=$ $\Delta^{-}=\bigcup_{i} \Delta_{i}^{-}$.

The points in $\Delta^{+} \cup \Gamma^{u}\left(\Delta^{-} \cup \Gamma^{s}\right)$ are those trapped in $C$ under backwards (forwards) iteration. Note that $\partial \Delta^{+} \subset \Gamma^{u}, \partial \Delta^{-} \subset \Gamma^{s}$ and that $\Delta^{-}, \Delta^{+}$are open subsets of $T$. It is not a priori true that the cusps of $\Delta^{-}$ are disjoint from those of $\Delta^{+}$.

\section{The noncompact leaves}

In this section we analyze the structure of a noncompact leaf of $\mathscr{F}$ and of the set of such leaves and then reduce the problem to a canonical case. In the end of the section we sketch the proof of the extension theorem for these leaves.

A noncompact leaf limits on compact leaves as follows [3]: Let $T$ be a depth one leaf limiting on a compact leaf $S$, say on the positive side. Let $W(S)$ be a tubular neighborhood of $S$ on the positive side. Then, if $W(S)$ is small enough, $D=T \cap W(S)$ covers $S$ infinite-to-one and the set of covering translations is a semigroup isomorphic to $\mathbf{N}$. There is a simple closed curve or disjoint union of properly embedded disjoint arcs $\delta \subset S$ called juncture so that $D=\bigcup_{i \geq 0} D_{i}$ and $D_{i}$ is homeomorphic to the closure of $S-\delta$. The lifts of $\delta$ to $D$ are also called junctures. Consecutive $D_{i}$ 's intersect along junctures.

Notice that once a leaf $T^{\prime}$ enters $W(S)$ it is forced to spiral towards $S$. Then except for a compact subsurface $T$ consists only of spiralling pieces. Unroll the spiralling to discover that $T$ is an end periodic surface.
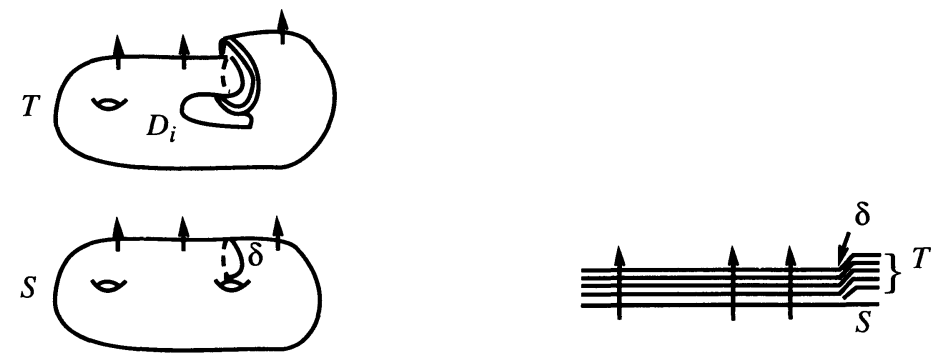

Figure 3. How $T$ SPIRALS TOWARDS A COMPACT LEAF. 
By the spiralling description it is easy to see that one can isotope the foliation (moving the points an arbitrarily small distance) to one which has smooth leaves and a smooth transversal vector field. This does not change limit point behavior.

If there are infinitely many compact leaves let $S_{n}$ be a sequence of distinct leaves. They limit on $S_{0}$ which by [9] is a compact leaf. The Reeb stability theorem implies that $S_{n}$ is isotopic to $S_{0}$ if it is close enough to it. This shows there are only finitely many isotopy classes of compact leaves of $\mathscr{F}$.

Define the equivalence relation $x \sim y$ if $x, y$ belong to the same leaf of $\mathscr{F}$. Branching occurs when there are $z_{n}, z, w_{n}, w$ with $z_{n} \rightarrow z$, $w_{n} \rightarrow w, z_{n} \sim w_{n}$, but $w \nsim z$.

Let $\mathscr{C}$ be the union of the compact leaves of $\mathscr{F}$ and $\mathscr{O}$ a component of $M-\mathscr{C}$. Let $T$ be a leaf contained in $\mathscr{O}$ so that $T$ spirals to $S \subset \mathscr{C}$ on the positive side of $S$. By the description above there is $W(S)$ where the spiralling $T \rightarrow S$ is occurring and every leaf intersecting $W(S)$ is not compact, therefore $(W(S)-S) \subset \mathscr{O}$. The spiralling description implies that the set of leaves intersecting $W(S)$ is topologically $S^{1}$. Since $W(S)$ is open, the union of these leaves, $\mathscr{O}^{\prime}$, is open in $M$. Let $x_{n} \in \mathscr{O}^{\prime}$, $x_{n} \rightarrow x$. Assume $x \notin \mathscr{C}$ so the leaf through $x$ spirals towards a compact leaf $S^{\prime}$ and let $\mathscr{O}^{\prime \prime}$ be the corresponding circle of leaves spiralling towards $S^{\prime}$. Since $x_{n} \rightarrow x, \mathscr{O}^{\prime} \cap \mathscr{O}^{\prime \prime} \neq \varnothing$ and is certainly an open set in $\mathscr{O}^{\prime}$ and $\mathscr{O}^{\prime \prime}$. If it is not closed then there is branching between the leaves of $\mathscr{O}^{\prime}$ and $\mathscr{O}^{\prime \prime}$ and this produces an infinite transversal segment in $\mathscr{O}^{\prime \prime} \cap \mathscr{O}^{\prime}$. As $M$ is compact this can be easily perturbed to a closed transversal in the intersection, which implies that $\mathscr{O}^{\prime \prime}=\mathscr{O}^{\prime}$. As a result, $x \in \mathscr{O}^{\prime}$ so $\mathscr{O}^{\prime}$ is also closed in $\mathscr{O}$. This shows:

Lemma 4.1. If $\mathscr{O}$ is a component of $M-\mathscr{C}$ then the foliation restricted to $\mathscr{O}$ is a fibration over $S^{1}$.

Fix a component $\mathscr{O}$ of $M-\mathscr{C}$ and $T$ a leaf of $\mathscr{F}$ in $\mathscr{O}$. The transversal flow induces a first return map in $T$ which is defined at least on the spiraling part of $T$. Let $f$ be this map.

Lemma 4.2. The transversal map is defined in all of $T$.

Proof. Let $x \in T$ so that the flow line through $x$ never hits $T$ again in positive time. The component $\mathscr{O}$ has compact closure and the flow line through $x$ does not exit this component, therefore this flow line limits on $y \in \mathscr{O}$. By a small perturbations of this flow segment a closed curve $\gamma \subset \mathscr{O}$ is produced, which is a transversal to $\mathscr{F}$ and does not intersect $T$. This is impossible because $\left.\mathscr{F}\right|_{\mathscr{O}}$ is a fibration with fiber $T$. q.e.d. 
Concluding, the local description shows that $T$ is an end periodic surface and the above lemma shows that $f$ is an end periodic homeomorphism.

We must first consider product components of $M-\mathscr{C}$. Let $E$ be a component of $M-\mathscr{C}$ so that $\partial E=S_{1} \cup S_{2}$, two isotopic compact leaves. Then $\bar{E}$ is homeomorphic to $S_{1} \times I$. Let $\delta$ be the juncture in $S_{1}$. Consider $D=\delta \times I$, an annulus embedded in $\bar{E}$. Using the techniques of Gabai [7, Theorem 7.10] one can isotope $D$ rel $\partial D$ to get an annulus which is transverse to $\mathscr{F}$ except for saddle type tangencies. As $\chi(D)=0$, there are no tangencies. Since $\delta$ is the juncture in $S_{1}$, the induced foliation in $D$ is a foliation by circles near $\delta \times\{0\}$ and since there is no holonomy restricted to the depth on leaves this is a foliation by circles in $D$. Cut $\bar{E}$ along $D$. The induced foliation has no holonomy near $S_{1}$. Again by Reeb stability the cut up foliation is a product foliation and the original foliation is obtained by glueing $\delta \times\{t\}$ in a copy of $D$ in the cut up manifold to $\delta \times\{h(t)\}$ in the other copy, where $h$ is a homeomorphism in $I$ fixing the endpoints and strictly monotone in the interior. In this case $T$ is just a bi-infinite stack of $S-\delta$ glued consecutively along junctures and the transversal map is just a shift by one in this stack. This is disallowed by the hypothesis because all monodromies of $\mathscr{F}$ in $M-\mathscr{C}$ are irreducible and consequently there are no product components.

We should mention that product components even though producing reducible monodromies would not present difficulties in terms of understanding the limit sets of the leaves. This occurs because in the universal cover $\widetilde{T}$ is isotopic to $\widetilde{S}_{1}$ by a bounded distortion isotopy and therefore will have the same limit set as $\widetilde{S}_{1}$, which is a Jordan curve.

Before attacking the extension problem we derive some properties of the limit sets of the depth one leaves. Let $Y$ be a depth one leaf in a component $Q$ of $M-\mathscr{C}$. The set $\partial Q$ is a finite union of closed leaves all of which are quasi-Fuchsian. Let $\widetilde{Q} \cong \widetilde{Y} \times \mathbf{R}$, a universal cover of $Q$ in $\widetilde{M}$, so that $\mathscr{F}$ corresponds to the product foliation in $\widetilde{Y} \times \mathbf{R}$. Denote by $L_{Y} \subset S_{\infty}^{2}$ the limit set of $\widetilde{Y} \times\{0\}$. Let $Y_{z}=\widetilde{Y} \times\{z\}$.

We first claim that $L_{Y} \neq S_{\infty}^{2}$. To see this, let $\widetilde{S} \subset \partial \widetilde{Q}$ and $L_{\widetilde{S}}$ be the Jordan curve in $S_{\infty}^{2}$ which is the limit set of $\widetilde{S}$. Its complement in $S_{\infty}^{2}$ is a union of two open disks $D_{1}, D_{2}$. Since $\tilde{S}$ separates $\mathbf{H}^{3}$ and $Y_{0}$ is contained in one component of the complement, $L_{\widetilde{Y}} \subset \bar{D}_{i}$ for some $i$.

Furthermore $L_{\widetilde{Y}}$ has empty interior. Let $V$ be an open set in $S_{\infty}^{2}$. Since $L_{\rho\left(\pi_{1}(M)\right)}=S_{\infty}^{2}$ (because $M$ is closed), there is $\alpha \in \pi_{1}(M)$ so that $E=\left(\rho(\alpha)\left(L_{\widetilde{S}}\right)\right)$ intersects $V$. As before $L_{\widetilde{Y}}$ is contained in the closure of 
one of the complementary components of $E$ (a Jordan curve) and cannot contain $V$.

On the other hand, as $Q$ is not a product, there are infinitely many boundary components of $\widetilde{Q}$, each generating a different quasicircle as limit set. The next result shows that $L_{Y}$ contains all of these curves and therefore cannot be a Jordan curve.

Lemma 4.3. $L_{Y} \supset L_{\widetilde{S}}$ for any $\widetilde{S} \subset \partial \widetilde{Q}$.

Proof. First notice that $L_{Y}=L_{Z}$ for any leaf $Z$ of the product foliation of $\widetilde{Q}$ because any two leaves are a bounded distance apart. This happens because any point in $Y_{0}$ is connected to a point in $Y_{1}$ by a transversal flow segment of bounded length. This is the key property used here.

Let $\widetilde{S} \subset \partial \widetilde{Q}$ and $\bar{\varphi}$ be the extension of $\varphi: \widetilde{S}=\mathbf{H}^{2} \rightarrow \mathbf{H}^{3}$ to $\mathbf{H}^{2} \cup S_{\infty}^{1}$. Let $x \in L_{\widetilde{S}}$ and $y \in S_{\infty}^{1}$ with $\bar{\varphi}(y)=x$, and let $V$ be a neighborhood of $y$ in $\mathbf{H}^{2} \cup S_{\infty}^{1}$. We show that $L_{Y} \cap \bar{\varphi}(V) \neq \varnothing$. Consider an oriented closed geodesic $\alpha$ in $S$ with basepoint $b$. By taking the inverse if necessary $\alpha$ has algebraic intersection number in $\geq 0$ with $\delta$, where $\delta$ is the juncture on $S$. Choose a lift $r \subset \widetilde{S}$ of $\alpha$ so that its positive limit points is in $V$. This is possible because $S$ is a closed hyperbolic surface and has $S_{\infty}^{1}$ as its limit set in $\mathbf{H}^{2} \cup S_{\infty}^{1}$. Fix $z \in \bar{\varphi}(r) \subset \widetilde{S}$. Move $z$ along the transversal flow to a nearby $Y_{n}$ for $n$ big. As in $\geq 0$ we can move $\bar{\varphi}(r)$ to a ray in $Y_{n}$, which is asymptotic to it if in $>0$, otherwise being a bounded distance from it. As $r$ is a geodesic ray on $\widetilde{S}$, its image in $\bar{\varphi}(\widetilde{S})$ has a unique limit point $x^{\prime} \in \bar{\varphi}(V)$, for $\rho\left(\pi_{1}(S)\right)$ is quasi-Fuchsian. The corresponding ray in $Y_{n}$ is either asymptotic to the first one or at most a bounded distance from it so it converges to $x^{\prime}$. Then $x^{\prime} \in L_{Y_{n}}=L_{Y}$. Therefore $L_{Y}$ is dense in $L_{\widetilde{S}}$ and being closed, contains it. q.e.d.

Some of these results could also be obtained more directly by nonelementary methods. Let $Q_{1}$ be the covering space of $M$ associated to $\pi_{1}(Q)$ and let $Q$ be embedded in it. Since $\partial Q$ is quasi-Fuchsian, $Q_{1}$ is geometrically finite [17, Chapter 8] and by Thurston [17, Theorem 8.12.4], $L_{Q_{1}}$ has measure 0 or 1 . Since $\pi_{1}(Y)$ is normal in $\pi_{1}(Q), L_{Y}=L_{Q}=L_{Q_{1}}$ and as $L_{Y} \neq S_{\infty}^{2}$ then its measure is zero. This also shows that $L_{Y}$ is the complement of a dense, countable union of open disks in $S_{\infty}^{2}$ (because $L_{Q_{1}}$ is) and therefore it is a Sierpinski curve. An open disk in question in one of the complementary components of $L_{\widetilde{S}}$, where $\widetilde{S} \subset \partial \widetilde{Q}$.

Each component of $\partial Q$ is a closed surface which implies that the corresponding juncture can and is from now on chosen to be a simple closed 

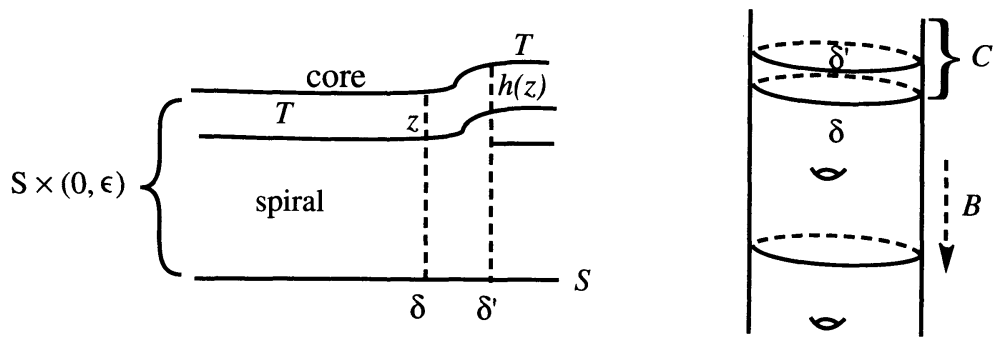

Figure 4. The FOLIATION IN The SPIRAL.

curve. The boundary of $Q$ consists of two sets $\partial_{+} Q$ and $\partial_{-} Q$, where the transversal vector field is outgoing and incoming respectively. At this point we can "forget" the rest of the manifold $\widetilde{M}$ because the boundary $\widetilde{Q}$ consists of quasidisks and the limit point behavior in $\widetilde{Q}$ is not affected by the rest of the manifold $\widetilde{M}$.

Let $\bar{Q}=Q \cup \partial Q$. Consider $S$ a component of $\partial \bar{Q}$ and a fixed tubular neighborhood $S \times(0, \varepsilon)$ of $S$ where the depth one leaves are spiralling towards $S$. Let $\delta$ be the juncture in $S$ and $G$ be a small annulus in $S$ with boundary $\delta \cup \delta^{\prime}$, where the spiralling direction is $\delta^{\prime} \rightarrow \delta . \delta, \delta^{\prime}$ also denote the lifts to $T$. Parametrize $S \times[0, \varepsilon]$ so that $\mathscr{F}$ is tangent to $S \times\{z\}$ outside of $G \times[0, \varepsilon]$ and in this set $\mathscr{F}$ is obtained by connecting $\delta \times\{z\}$ to $\delta \times\{h(z)\}$, where $h$ is the germ of a homeomorphism of the interval, with $h(z)>z \forall z \neq 0$ (see Figure 4). We choose the neighborhood $S \times(0, \varepsilon)$ to be of this type to start with. The set $S \times(0, \varepsilon)=s p_{S}$ is the spiral associated to $S$. The union of the spiralling sets in the spiral (=sp) of $Q$. The complement is the core of $Q$. Notice that the core is compact and disjoint from $\partial Q$.

We will need the fact that $Q$ is convex later on. Since $Q_{1}$ is geometrically finite, its convex hull is compact [17] and is isotopic to the closure of $Q$. Isotope $Q$ to $N$, which contains the convex hull of $Q_{1}$. Isotope the foliation producing a foliation in $N$ with corresponding core, spirals and ladders. The new foliation will be denoted by $\mathscr{F}$ also. We may also assume $\operatorname{core}(N) \subset$ convex $\operatorname{hull}\left(Q_{1}\right)$. The isotopy moves every point a bounded distance so limit point behavior is not affected. We denote the core of $N(\widetilde{N})$ by $C(N),(C(\widetilde{N}))$. The lift of the spiral to $\widetilde{N}$ is also called the spiral. The only compact leaves of $\mathscr{F}$ are the boundary leaves which correspond to quasi-Fuchsian groups and $\mathscr{F}$ is a fibration 
in the interior. The monodromy associated to the depth one leaves is an irreducible end periodic map. The junctures are simple closed curves in $\partial N$. The boundary of the core of $N$ is $\partial N \times\{\varepsilon\}$. Let $\pi: \widetilde{N} \rightarrow N$ be the covering map. This induces a covering map $\pi: \widetilde{T} \times\{0\} \rightarrow T$.

The limit set of $\widetilde{T}$ is independent of the leaf $T$ in the interior of $N$. From now on fix one such leaf $T$. We usually identify $\widetilde{T}$ to $\widetilde{T} \times\{0\} \subset \widetilde{N}$. The core of $T$ is the closure $C$ of the intersection of $T$ and the interior of the core of $N$. Then $\partial C$ is a union of junctures. The positive ladder of $T$ is denoted by $B$, the negative by $A$. The monodromy of the fibration is $f$. First imbed $T$ with a hyperbolic metric. As opposed to the case for compact leaves, not all hyperbolic metrics are quasiconformal. It is natural to find one which is quasiconformal with the induced metric from $N$. Fix a hyperbolic structure on $\partial N$ for which the junctures are geodesic in the respective components. Lift these structures to the ladder part of $T$ by the pullback of the projection (using the transversal flow). Choose any hyperbolic structure on the core of $T$ which matches the lengths of the junctures in $\partial C$. In the ladder, $f$ is an isometry. We fix this hyperbolic structure on $T$ once and for all, and denote it by $d_{T}$ either in $T$ or in $\widetilde{T}$. Then $\widetilde{T}$ is isometric to $\mathbf{H}^{2}$ and it has a well-defined circle at infinity, $S_{\infty}^{1}$. The circle at infinity is an invariant of the quasi-isometry type of the metric on $\widetilde{T}$ and therefore depends only on the metric on $N$.

Let $\varphi: T \rightarrow N$ be the embedding of $T$ into $N$ and choose a lift $\varphi_{\widetilde{T}}: \widetilde{T}=\mathbf{H}^{2} \rightarrow \widetilde{N} \subset \mathbf{H}^{3}$. We want to extend $\varphi_{\widetilde{T}}$ to $S_{\infty}^{1}$ continuously. The first observation is that $\varphi_{\widetilde{T}}$ cannot be a quasi-isometry, for then $L_{\widetilde{T}}$ would be a Jordan curve, a contradiction. If there is a continuous extension, there will be pinching: a geodesic in $\widetilde{T}$ which is mapped to a curve in $\mathbf{H}^{3}$ with both limit points identified.

The general idea of the proof is the following: Let $\Gamma^{s}$ and $\Gamma^{u}$ be the invariant laminations provided by the end periodic theory. In the hyperbolic metric on $\widetilde{T}$ let $p$ be an ideal point and $r$ a geodesic ray defining it. If $r$ keeps crossing $\Gamma^{s} \cup \Gamma^{u}$ then these leaves bound sets which form a neighborhood basis for $p$ in $\mathbf{H}^{2}$ (see Figure 5(a), next page). Otherwise $r$ will be contained in the ladder so $\varphi_{\widetilde{T}}(r)$ projects to $\partial \widetilde{N}$ and will have a well-defined limit point (because $\partial N$ is quasi-Fuchsian).

In the first case the strategy is to produce a neighborhood basis in the closure $N^{*}$ of $\widetilde{N}$ in $\mathbf{H}^{3} \cup S_{\infty}^{2}$ for the eventual limit point of $\varphi_{\widetilde{T}}(r)$ in $S_{\infty}^{2}$. In $\tilde{N} \cong \widetilde{T} \times \mathbf{R}$ consider the laminations $\Gamma^{u} \times \mathbf{R}$ and $\Gamma^{s} \times \mathbf{R}$. Any leaf of one of these laminations separates $\widetilde{N}$ and after they are "capped off" they will separate $\mathbf{H}^{3}$. This operation is explained in the last section. 


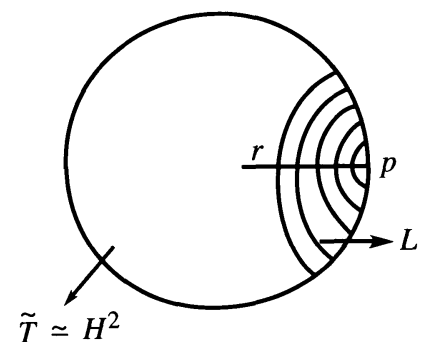

(a)

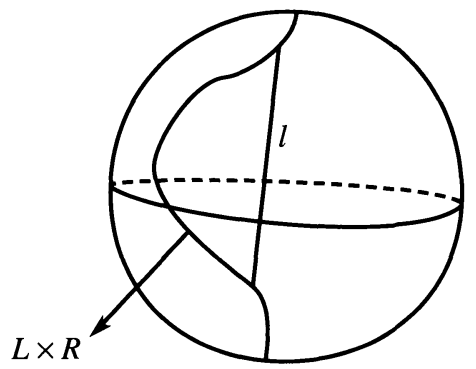

(b)

Figure 5. (a) NeIgHborhood BASIS; (b) BENT LEAF.

Then they will bound a neighborhood basis of a point in $S_{\infty}^{2}$ defining the limit point of $\varphi_{\widetilde{T}}(r)$. This idea is an extension of the technique used by Cannon and Thurston [2] in the case of a fibration. To achieve this the sets $L \times \mathbf{R}$, where $L$ is a leaf of one of the laminations, have to be essentially geodesic-otherwise they may not define a neighborhood basis as indicated in Figure 5(b). The property needed is that $L \times \mathbf{R}$ is uniformly quasigeodesic in $\mathbf{H}^{3}$. Then hyperbolic geodesics in $\mathbf{H}^{3}$ connecting points in this set do not stray very far from it, which will imply that as the $L_{n} \times \mathbf{R}$ move to infinity they will "shrink" and satisfy the property required.

The biggest difficulty is to understand the geometry of $L \times \mathbf{R}$ in the hyperbolic metric. The solution will be to construct a "distance" function where we understand that distances as related to the foliation and the properties of $L \times \mathbf{R}$ are clearer, while at the same time it is not too distorted with respect to the hyperbolice metric, that is, quasi-isometric to it. Irreducibility of the monodromy will be essential to show this fact.

\section{Quasi-isometry of semimetrics}

As the end periodic monodromy $f: T \rightarrow T$ is irreducible is isotopic to a map $g$, given by the end periodic theory, which satisfies the properties described in $\S 3$. This induces a new transversal flow in $N$ called the $g$-flow. The original flow in $N$, which (in our case) comes from a flow in $M$, is the $f$-flow. Notice $N$ is an open manifold and $\bar{N}$ is its compactification.

We first install projectively invariant transverse measures to the invariant laminations in $T$. Let $\eta_{i}, 1 \leq i \leq n^{\prime}$, be the nonescaping arcs associated to $\Gamma^{u}, \eta_{i} \subset C$ and $\partial \eta_{i} \subset \partial_{+} C$. Then $g\left(\eta_{j}\right)$ intersects $C$ in 
finitely many arcs isotopic to $\eta_{i}$. Let $p_{i j}$ be the number of components of $g\left(\eta_{j}\right) \cap C$ isotopic rel $\partial_{+} C$ to $\eta_{i}$. This produces an intersection matrix $P=\left(p_{i j}\right)$. When there is a positive eigenvector to this matrix the transverse measure produced will have full support. Given this hypothesis we proved the extension theorem in [4]. Unfortunately, as shown in [5], this is note always the case, and as a matter of fact the existence of such measures in the nongeneric case. The reason for this is that the invariant laminations for the end periodic homeomorphism are produced through a nonmixing process, that is, as the limit of a sequence of disjoint geodesic simple closed curves or arcs as opposed to the finite genus case. It is our feeling that the construction described below and not the one associated to the full support case is the most natural for depth one foliations because of the nonmixing property. Let

$$
H=\max _{1 \leq i \leq n^{\prime}}\left(\sum_{1 \leq j \leq n^{\prime}} p_{i j}\right) .
$$

Fix $n_{0}>0$ so that $\forall n \geq n_{0}, g^{n}\left(\partial_{-} C\right) \cap C$ only consists of arcs isotopic rel $\partial_{+} C$ to the $\eta_{i}$. Let $m^{\prime}$ be the maximum in $i$ and $n, 1 \leq n \leq n_{0}$, of the number of strands of $g^{n}\left(\partial_{-} C\right) \cap C$ isotopic to $\eta_{i}$ rel $\partial_{+} C$. Let $\lambda=2 m^{\prime} H$.

Put the atomic measure $\lambda^{-n}$ across $g^{n}\left(\partial_{-} C\right)$. As $g^{n}\left(\partial_{-} C\right) \rightarrow \Gamma^{u}$ and all atomic measures are nonzero, the support of the final measure is $V_{+}$.

Proposition 5.1. The measure above defines a transverse measure to $V_{+}$.

Proof. We have to show it does not blow up, that is, measures of finite transverse arcs are finite. Since $g^{n}\left(\partial_{-} C\right)$ only accumulates in $\Gamma^{u}$, we only have to prove the measure does not blow up in $\Gamma^{u}$. This is done by showing that the transverse measures to all the bands of arcs isotopic to $\eta_{i}$ rel $\partial_{+} C$ are finite. Let $F_{i} \quad 1 \leq i \leq n^{\prime}$, be these bands.

Since there are at most $m^{\prime}$ strands of $g^{n}\left(\partial_{-} C\right) \cap C$ isotopic to any $\eta_{i}$, the transverse measure to $F_{i}$ obtained from $g\left(\partial_{-} C\right) \cap C$ is $\leq 1 / 2$. The measure in $F_{i}$ coming from $g^{2}\left(\partial_{-} C\right) \cap C$ is smaller than

$$
m^{\prime} \frac{1}{2 m^{\prime} H} \frac{1}{2}<\frac{1}{4} \text {. }
$$

By induction the measure in $F_{i}$ coming from $g^{n}\left(\partial_{-} C\right) \cap C$ is $<1 / 2^{n}$ for $n \leq n_{0}$. Then in step $n_{0}+1$ the measure added across $F_{i}$ is

$$
m_{i}^{\prime}=\frac{1}{2 m^{\prime} H} \sum_{j=1}^{n^{\prime}} p_{i j} m_{j},
$$


where $m_{j}$ is the measure in $F_{j}$ coming from $g^{n_{0}}\left(\partial_{-} C\right) \cap C$. Since $m_{j} \leq$ $1 / 2^{n_{0}}$ for all $j$ :

$$
m_{i}^{\prime} \leq \frac{1}{2^{n_{0}}} \frac{1}{2 m^{\prime} H}\left(\sum_{j=1}^{n^{\prime}} p_{i j}\right) \leq \frac{H}{2^{n_{0}}\left(2 m^{\prime} H\right)}<\frac{1}{2^{n_{0}+1}} .
$$

The measure in $F_{i}$ arising from $g^{n_{0}+2}\left(\partial_{-} C\right) \cap C$ comes from one iteration of $g^{n_{0}+1}\left(\partial_{-} C\right) \cap C$ and so this added measure is $\leq 1 / 2^{n_{0}+2}$. By induction the total measure across the band is $\leq 1$.

This shows the measure does not blow up in $\Gamma^{u} \cap C$. Given any other point $y \in \Gamma^{u}$, let $x \in \Gamma^{u} \cap C$ be a point in the same leaf. As $g^{n}\left(\partial_{-} C\right)$ is geodesic, it is very near $x$ if and only if it is very near $y$. Therefore the transverse measure to a small transverse arc at $y$ is finite. q.e.d.

We denote this transverse measure by $d y$. It is projectively invariant under $g$ with factor $\lambda$. The difference from the full support ("pseudoAnosov") case is that the support of the measure in that case is $\Gamma^{u}$ while here it is $V_{+}=\Gamma^{u} \cup W_{+}$. Furthermore, if there is a projectively invariant transverse measure with expansion factor $\lambda^{\prime}$ and support contained in $\Gamma^{u}$ then $\lambda^{\prime} \leq \lambda$, so the eigenvalues of $P$ give the smallest expansion factors. In the full support case the eigenvalue produced is the best possible. All of this shows some important differences between irreducible end periodic homeomorphisms and irreducible or pseudo-Anosov homeomorphisms of finite genus surfaces.

Do the same construction for $\Gamma^{\mathcal{S}}=\lim _{n \rightarrow-\infty} g^{n}\left(\partial_{+} C\right)$ yielding the transverse measure $d x$ to $V_{-}$. Then $d x+d y$ produces a path semimetric in $T$ for which the distance between two points is the minimum of the lengths of paths connecting them, where the length of a path $\gamma$ is $\int_{y} d x+d y$.

We will call $d x+d y$ an infinitesimal semimetric. Notice that infinitesimal here is interpreted in a generalized sense: there are atomic measures across some leaves and if a path crosses such a leaf it carries the full atomic measure no matter how small it is. However if it has an endpoint in such a leaf it does not carry any of the atomic measure. This convention will be used throughout the rest of the article.

The distance function defined in $T$ is obviously not a metric because any two points in a component of $T-\left(\Gamma^{u} \cup \Gamma^{s}\right)$ are joined by a zero length path. The atoms of $d x$ or $d y$ keep this from even being a pseudometric: it would not satisfy the triangle inequality $d(x, y) \leq d(x, z)+d(z, y)$ when $z$ is in an atomic leaf, because that atomic measure is not present in the right side of the inequality. We will use a modified version of $d$ where 
the atomic measures are bounded. Take $\theta>$ maximum of atomic measures. Then for any $x, y, z, d(x, y) \leq d(x, z)+d(z, y)+\theta$. Therefore $d$ is a semimetric.

We identify $\widetilde{N}$ to $\widetilde{T} \times \mathbf{R}$, where $\widetilde{T} \times\{\cdot\}$ is a leaf of the foliation $\widetilde{F}$ in $\tilde{N}$ and $\{\cdot\} \times \mathbf{R}$ is a $g$-flow line. We may assume the expansion and contraction factors of $d x$ and $d y$, respectively, are the same by choosing the biggest of them in both constructions. Lift $d x, d y$ to $\widetilde{T} \times\{0\}$, also denoted by $d x, d y$. Install the infinitesimal semimetric $d s_{0}$ in $\widetilde{N}$ :

$$
d s_{0}=\lambda^{t} d x+\lambda^{-t} d y+|d t|
$$

where $t$ is the vertical coordinate in $\widetilde{T} \times \mathbf{R}$. By the projective invariance of $d x$ and $d y, d s_{0}$ is invariant under covering translations of $N$ and induces an infinitesimal semimetric in $N$.

Let $d_{H}$ be the hyperbolic metric in $N$ or $\tilde{N}$. Our goal is to show that the two semimetrics are quasi-isometric in $\tilde{N}$. This is not possible yet because of two reasons:

First problem. The $d t$ factor is a big distortion of the hyperbolic length near $\partial N$. Take a small transverse segment to $\mathscr{F}$ with one endpoint in $\partial_{+} N$. It has a finite hyperbolic length but it will cross $T$ infinitely (since $T$ limits on $\partial N$ ) and will therefore have infinite $d$-length. In fact any point in $\partial N$ required infinite $d$-length to be achieved as opposed to finite hyperbolic length. Let $h_{+}\left(h_{-}\right)$be the function in $N$ which is 0 in the positive (negative) spiral and 1 everywhere else. The same notation will be used for the lifts to $\widetilde{N}$. Change the factor $|d t|$ in $\widetilde{N}$ to $h_{-} h_{+}|d t|$.

Second problem. The part of $d s$ tangent to $\mathscr{F}$ is not good near $\partial N$ either. Here the opposite behavior from the first problem occurs: the hyperbolic length might be very big and the semimetric length quite small. Let $\alpha \subset T$ be a closed geodesic contained in (say) the positive ladder. Let $\mathrm{pr}_{+}$be the projection to $\partial_{+} N$. If $\alpha$ is very near $\partial_{+} N, l_{H}(\alpha)$ is very near $l_{H}\left(\operatorname{pr}_{+}(\alpha)\right)$ and in fact $l_{H}\left(g^{n}(\alpha)\right)$ stabilizes as $n \rightarrow \infty$ (see description below). On the other hand, $\alpha$ does not cross $\Gamma^{\mathcal{S}}$ (as $\alpha$ is contained in the positive ladder) and $\alpha$ contained in a leaf of $\mathscr{F}$ implies that $l(\alpha)$ comes only from the integral of the $d y$ measure and so $l\left(g^{n}(\alpha)\right)<\lambda^{-n}(l(\alpha))$. By taking $m$ big enough, $l_{H}\left(g^{n}\left(\alpha^{m}\right)\right)$ is comparable to $m l_{H}(\alpha)$ which is very big but the $d$-length being $\left\langle\lambda^{-n}\left(l\left(\alpha^{m}\right)\right)\right.$ can be very small if $n \gg m$, contradicting quasicomparability.

The key to solve this is to destroy the exponential collapsing near $\partial N$. Let $d s_{s}$ be the infinitesimal semimetric induced by $d s_{0}$ in $\partial N \times\{\varepsilon\}$. Induce an infinitesimal semimetric in $\partial_{+} N \times(0, \varepsilon]$ by the pullback of 
$d s_{s}$ under the projection to $\partial_{+} N \times\{\varepsilon\}$ along $g$-flow lines. This will be denoted by $d s_{s}$ either in $N$ or $\tilde{N}$. Do the same for $\partial_{-} N$. Define the infinitesimal semimetric $d s$ in $\widetilde{N}$ :

$$
d s=h_{+} \lambda^{t} d x+h_{-} \lambda^{-t} d y+h_{+} h_{-}|d t|+\left(1-h_{+} h_{-}\right) d s_{s} .
$$

As before $d s$ is invariant under covering translations so it defines an infinitesimal semimetric in $N$. Let $d$ be the path integral of $d s$ in $N$ or $\widetilde{N}$ and $l$ the length function associated to it. Notice that all $h_{+} \lambda^{t} d x$ does is to eliminate the blow up on positive iterates of the positive juncture and therefore the atomic measures are bounded. Therefore $\left(V_{-}, h_{+} d x\right)$ is a measured lamination in $T$.

By multiplying the original measures $d x, d y$ by a constant smaller than 1 we can assume all atomic measures of $V_{+}, V_{-}$are bounded above by $b=1 / 2$. As before $d$ is a semimetric, where we can take $\theta=1 / 2$.

The map which fixes the core of $N$ and in the spiral projects a point along its $g$-flow line (in the positive direction if the point is in the negative spiral and negative if on the other spiral) to the first intersection with $\partial N \times\{\varepsilon\}$ is the push off map and is denoted by $p_{1}$. It moves points in $N$ away from $\partial N$. It is obviously a continuous map. Its lift to $\widetilde{N}$ is also called push off and also denoted by $p_{1}$.

Proposition 5.2. The push off map is a d-distance decreasing retraction $p_{1}: N \rightarrow C(N)$.

Proof. Let $\gamma^{\prime}$ be a path in $N$ and $\gamma$ a component of its intersection with the spiral, say in $S \times(0, \varepsilon)$. Then $p_{1}(\gamma) \subset S \times\{\varepsilon\}$ and by the definition of the semimetric $d, \int_{\gamma} d s_{s}=l\left(p_{1}(\gamma)\right)$. But $l(\gamma) \geq \int_{\gamma} d s_{s}$ so $l(\gamma) \geq l\left(p_{1}(\gamma)\right)$. The components of $\gamma^{\prime} \cap C(N)$ are kept fixed and so have length preserved by $p_{1}$. Since $d$ is a path semimetric the result follows. q.e.d.

There are a couple of subtle points associated to the new flow in $N$. The map $f$ is an isometry (in the hyperbolic metric) in the ladders of $T$ but, as the next result shows, in general $g$ cannot satisfy this property. On the other hand, $g$ preserves the geodesic laminations $\Gamma^{u}, \Gamma^{s}, V_{-}, V_{+}$ and $f$ does not.

Every leaf of $\Gamma^{u}$ intersects $\Gamma^{s}$ transversely. An escaping ray of $\Gamma^{u}$ is an infinite component of $\Gamma^{u}-\Gamma^{s}$. In [5] we show that an escaping ray is periodic under $g$. Furthermore there are only finitely many escaping rays. Every other component of $\Gamma^{u}-\Gamma^{s}$ is a finite segment with both endpoints in $\Gamma^{s}$.

Proposition 5.3 [5]. Let $S$ be a component of $\partial_{+} N$. The lamination $\Gamma^{u} \downarrow S_{\varepsilon}=\pi\left(\Gamma^{u} \times \mathbf{R}\right) \cap S_{\varepsilon}$ in $S_{\varepsilon}$ has finitely many closed leaves, each of 
which corresponds to an escaping ray of $\Gamma^{u}$ in $T$. The other leaves (if any) are grouped into finitely many bands of isotopic leaves which jointly spiral towards closed leaves in both directions. In general this lamination is not geodesic. When $g$ (or $f$ for that matter) is irreducible and $\partial T=\varnothing$ this lamination fills $S_{\varepsilon}$ (in the sense that complementary regions are simply connected) except for possible annuli in between isotopic closed leaves. Similar results hold for $\Gamma^{s}, V_{-}, V_{+}$.

In general the bands above from a Cantor set of leaves (see [4]). The arguments in [5] show that the spiralling of $l^{\prime}$ to $l$ in $S_{\varepsilon}$ corresponds in $T$ to the boundary of the lift of $l^{\prime}$ to $T$ (a finite segment with endpoints in $\Gamma^{s}$ ). It is opposite to the escaping direction of the lift of $l$ to $T$ which goes deeper and deeper into the ladder.

An atomic leaf $l$ of $\Gamma^{s} \cup \Gamma^{u}$ cannot limit in $C$, therefore it contains two escaping rays and is periodic. As a result there are at most finitely many atomic leaves.

Another important question in our analysis is what happens near $\partial N$. The $g$-flow, unlike the $f$-flow, is only defined in $N$ and there are obstructions to extending it to a semiflow in $\bar{N}$.

Proposition 5.4 [5]. There is a map $g$ given by the end periodic theory satisfying the following: Let $S$ be a component of $\partial_{+} N$. If $x \in S_{\varepsilon}$ then the $g$-flow line through $x$ converges in positive time to a point $\phi_{+}(x)$ in $S$. The hyperbolic length of the $g$-flow ray between $x$ and $\phi_{+}(x)$ has bounded length and the function $\phi_{+}: S_{\varepsilon} \rightarrow S$ is continuous. Obviously there is also a g-flow projection $S \times\left\{\varepsilon^{\prime}\right\} \rightarrow S$ for $\varepsilon^{\prime}<\varepsilon$. This projection gets uniformly close to the f-flow projection when $\varepsilon^{\prime} \rightarrow 0$.

This is not true for all $g$ preserving the laminations because $g$ was only determined in $V^{+} \cup V^{-}$and there is freedom in the complementary regions. A map $g$ satisfying the above properties is called a tight representative. From now on fix one tight $g$, which we can assume was the original one.

The extended flow segment through $x \in S_{\varepsilon}$ is transverse to $\partial_{+} N$. Unfortunately the projection $\phi_{+}$is not a homeomorphism because in general infinitely many flow segments will collapse to a single point in $\partial_{+} N$ (see [5]). Therefore the $g$-flow cannot extend to a semiflow in $\bar{N}$. Let $\Lambda^{u}$ be the geodesic lamination in $S_{\varepsilon}$ homotopic to $\Gamma^{u} \downarrow S_{\varepsilon}$ (induce a hyperbolic metric in $S_{\varepsilon}$ by the pullback of the projection to $S$ under the $f$-flow).

Proposition 5.5 [5]. The projection $\Gamma^{u} \downarrow S$ of $\Gamma^{u} \downarrow S_{\varepsilon}$ to $S$ under the $g$-flow is a geodesic lamination equal to the projection of $\Lambda^{u}$ to $S$ under the $f$-flow. It has finitely many leaves and if $g$ is irreducible and $\partial T=\varnothing$ 
then it fills $S$. Isotopic leaves of $\Gamma^{u} \downarrow S_{\varepsilon}$ are identified under the projection and all identifications are a result of the collapsing of isotopic leaves.

Given $S \subset \partial N$ let $\delta_{S}$ be the corresponding juncture, and $G_{S}$ the corresponding juncture annulus where $S_{\varepsilon}$ is not tangent to the foliation. Notice $|d t|$ in $S_{\varepsilon}$ is nonzero exactly in $G_{S}$. Furthermore, any transversal segment to $G_{S}$ has $|d t|$-length $\geq 1$. This happens because across $G_{S}$ we move one step down (or up) in the ladder. The set $J_{S}=\left(p_{1}\right)^{-1}\left(G_{S}\right)$ is a solid torus in $N$. This set is called a juncture barrier as is the union of these over all $S \subset \partial N$. There are finitely many of these solid tori in $N$ and they are disjoint. A lift of one of the barriers to $\widetilde{N}$ is an infinite thickened band with the lower boundary in $\partial \widetilde{N}$ the upper boundary in $C(\widetilde{N})$ and lateral boundaries consisting of $g$-flow segments. It is also called a juncture barrier. The $d s_{s}$ part of the measures associated to $|d t|$ occur exactly in the juncture barriers because $G_{S} \times\{\varepsilon\}$ is exactly where $S_{\varepsilon}$ is not tangent to the foliation producing $|d t|$-length. They are called the juncture measures.

We proceed to show that $d$ and $d_{H}$ are quasi-isometric on $\tilde{N}$. The first step is to show that the induced semimetrics are quasicomparable in the leaves. Proving the result for the leaf $T$ implies the result for all leaves. Let $d^{\prime}$ be the semimetric $d$ restricted to $T$ or $\widetilde{T}$.

Theorem 5.6. The semimetric $d^{\prime}$ and the hyperbolic metric $d_{T}$ are quasi-isometric in $\widetilde{T}$.

Proof. First we show that there is $\tau>0$ satisfying: if $\alpha$ is a hyperbolic geodesic segment in $T$ of length 1 , then $l(\alpha) \leq \tau$. If $\alpha$ is contained in the core then this is true because the measures are finite for each arc and $C$ is compact. This can be extended to any compact subsurface of $T$ because the added transversal measures to $V_{-}, V_{+}$, and the juncture annuli are all finite.

It remains to see what happens when $\alpha$ is deep in the ladder. By Proposition 5.4 such a segment projects to a segment in $\partial N$ by the $g$ flow which is very close to being geodesic and has length approximated by 1. Therefore it has a bounded measure in $S$ (the measure induced by the projection of the $g$-flow). This measure is $\leq$ twice the $d s_{s}$-length of $\alpha$ so the result follows.

Let now $p, q$ be any two points in $\widetilde{T}$ and join them by a hyperbolic geodesic arc $\gamma$ broken into $\gamma_{0}, \cdots, \gamma_{m}$, where $l_{T}\left(\gamma_{i}\right)=1,1 \leq i \leq m$ and $0 \leq l_{T}\left(\gamma_{0}\right)<1$. Then $l\left(\gamma_{i}\right) \leq \tau$. There might be atoms in between so $l(\gamma) \leq(m+1) \tau+m b=m(b+\tau)+b$. But $m \leq l_{T}(\gamma)=d_{T}(p, q)$ so $d^{\prime}(p, q) \leq l(\gamma) \leq d_{T}(p, q)(b+\tau)+\tau$. The other required inequality for quasicomparability is harder to prove and needs a preliminary result. 
Proposition 5.7. Let $R$ be a compact hyperbolic surface either closed or with geodesic boundary and let $\Gamma_{1}, \Gamma_{2}$ be properly embedded transverse geodesic laminations with transverse measures of full support. Suppose that their union binds $R$, meaning that complementary regions of $\Gamma_{1} \cup \Gamma_{2}$ have compact closure and are simply connected. Then the hyperbolic metric on $\widetilde{R}$ is quasi-isometric to the semimetric coming from integration of the transverse measures.

Proof. Let $d_{h}$ be the hyperbolic metric in $R$ or $\widetilde{R}$, and $d$ the path semimetric. As shown above there are $b_{1}, b_{2}>0$ so that $d(p, q)<$ $b_{1} d_{h}(p, q)+b_{2}$ for any $p, q \in \widetilde{R}$.

Claim 1. There are $\mu>0, \nu>1, \xi>0$ so that any hyperbolic segment of length $>\nu$ crosses at least one of the laminations at an angle $>\xi>0$. Furthermore there is a subsegment of length $\geq \mu$ where such a crossing occurs in midpoint.

Suppose there is a sequence of geodesic arcs of length $>n, n \in \mathbf{N}$, crossing the laminations, if at all, only at angles $\leq 1 / n$. Project to $R$. Take a limit point in $R$ of the midpoints of the arcs and a limiting direction of the arcs. It follows that the geodesic through this point with this direction does not cross either lamination, contradicting the fact that they bind $R$. This shows there are $\mu, \xi>0$ so that each geodesic arc of length at least $\mu$ crosses at least one of the laminations at an angle $>\xi$. Take $\nu=3 \mu$.

Claim 2. There is $\mu^{\prime}>0$ so that segments as above have measure at least $\mu^{\prime}$.

Since any such segment crosses $\Gamma_{1} \cup \Gamma_{2}$ it has a positive measure. If the claim is not true find a sequence of arcs $\gamma_{n}$ of length $=\mu$ crossing, say, $\Gamma_{1}$ in the midpoint. Project to $R$. Take a subsequence where the midpoints and directions converge. The limit point is in $\Gamma_{1}$ (a closed subset of $R$ ) and the limit segment $\gamma$ makes an angle $\geq \xi$ with $\Gamma_{1}$ and therefore its middle half has measure $z>0$. Any sufficiently close segment will have to transversally intersect at least these leaves, so the claim follows.

By Claim 2 there are $\mu^{\prime}, \nu>0$ so that any hyperbolic segment of length $>\nu$ has at least measure $\mu^{\prime}$. Take $K^{\prime}=2 \nu$ and $k^{\prime}=2 \nu / \mu^{\prime}$. Let $\gamma$ be the hyperbolic geodesic segment in $\widetilde{R}$ connecting $p, q$ with $d_{h}$-length $l$ and let $r$ be its total transverse measure. If $l>K^{\prime}$ then

$$
l / \nu>l / \nu-1>l / K^{\prime} .
$$

There are at least $l / \nu-1$ subsegments of $d_{h}$-length $\geq \nu$, each with measure at least $\mu^{\prime}$, so the total measure of the segment is

$$
r \geq \mu^{\prime}(l / \nu-1)>\mu^{\prime}(l / 2 \nu)=l / k^{\prime} .
$$


Since $d_{h}(p, q)=l$, it follows that $d_{h}(p, q)<k^{\prime} r$. The laminations being geodesic imply that the most efficient paths in terms of the transversal measures are the hyperbolic geodesic ones, so $r=d(p, q)$. Therefore $d_{h}(p, q)<k^{\prime} d(p, q)+K^{\prime}$ and quasicomparability follows. q.e.d.

Continuation of the proof of Theorem 5.6. The original projectively invariant transverse measure $d y$ to the lamination $\pi\left(V_{+} \times \mathbf{R}\right)$ is eliminated in the negative ladder by $h_{-}$. Then $d s_{s}$ is added in $\partial_{-} N \times(0, \varepsilon)$ and this is constant across $\delta \times(0, \varepsilon]$, where $\delta$ is the union of the negative junctures. This produces a transverse measure to $\pi\left(V_{+} \times \mathbf{R}\right)$ with full support, which is constant in the negative spiral and changes by $\lambda^{-\Delta t}$ everywhere else. It is called the basic measure and it does not include the $d s_{s}$ factor transverse to $V_{+} \times \mathbf{R}$ in the positive spiral or the $|d t|$ factor. These factors are called the remaining $d s_{s}$ factors. The same applies to $d x$.

Claim. There is $c>0$ so that for any $p, q \in \widetilde{T}, d^{\prime}(p, q) \leq 1 \Rightarrow$ $d_{T}(p, q)<c$.

Note this is not the same as the previous proposition because $T$ is an infinite genus surface. Choose a path $\gamma$ connecting $p, q$ with $l(\gamma)<$ 1.2. Then it crosses less than three juncture annuli. We will again use Proposition 5.4: even though $g$ is not an isometry it is uniformly near an isometry deep in the ladders. The $g$-flow lines stabilize uniformly near $\partial N$, therefore given $\tau_{1}>0$ we can find $\tau_{2}>0$ so that

$$
d_{H}(x, \partial N)<\tau_{2} \Rightarrow d_{H}\left(f^{-n} g^{n}(x), x\right)<\tau_{1} \quad \forall n \geq 0, x \in T .
$$

Choose $n^{\prime}$ big enough so that every point in $\bigcup_{i>n^{\prime}}\left(B_{i} \cup A_{i}\right)$ is $\tau_{2}$ near $\partial N$ and choose $c^{\prime}$ which satisfies the claim for

$$
R=C \cup\left(\bigcup_{i=0}^{n^{\prime}+2}\left(B_{i} \cup A_{n}\right)\right) \text {. }
$$

This is possible because $\left(V_{+} \cup V_{-}\right) \cap R$ is a union of transverse geodesic laminations which bind $R$ and the basic transverse measures to these laminations have full support, so we can apply the previous proposition.

When $\gamma \cap\left(C \cup\left(\bigcup_{i=0}^{n^{\prime}} A_{i} \cup B_{i}\right)\right) \neq \varnothing$ the above proof works. Otherwise $\gamma$ is deep in one of the ladders, say $B$. Flow $\gamma$ backwards by some iterate of $g^{-1}$, say $g^{-s}$, sending it to $\left(B_{n^{\prime}} \cup B_{n^{\prime}+1} \cup B_{n^{\prime}+2}\right)$. The $d^{\prime}$-length of $g^{-s}(\gamma)$ (equal to its $d$-length) is $<2 l(\gamma)<4$ because $d$-length is bounded by twice the $d s_{s}$-length in the spiral. Therefore by the result for $R$ there is $c_{1}>0$ fixed so that the endpoints of $g^{-s}(\gamma)$ are connected by a 
hyperbolic geodesic arc $\gamma^{\prime}$ of length $<c_{1}$. The remark above implies that the hyperbolic geodesic arc homotopic to $g^{s}\left(\gamma^{\prime}\right)$ (which is also homotopic to $\gamma$ ) has length $<c_{1}+2 \tau_{1}$ and the claim follows.

Given any two points $p, q \in \widetilde{T}$ choose a path $\gamma$ connecting them with $l(\gamma)<d^{\prime}(p, q)+1 / 2$. Break it into at most $2\left(d^{\prime}(p, q)+1\right)$ subpaths $\gamma_{i}$ satisfying $1 / 2<l\left(\gamma_{i}\right)<1$, which is possible since the maximum of the atomic measures is $=1 / 2$. Choose the endpoints $p_{i}, q_{i}$ to be outside $V_{+} \cup V_{-}$. By the above claim $d_{T}\left(p_{i}, q_{i}\right)<c$ so $d_{T}(p, q)<2 c\left(d^{\prime}(p, q)+1\right)$, which completes the proof.

Theorem 5.8. $\quad d$ and $d_{H}$ are quasi-isometric in $\tilde{N}$.

Proof. $d$ on $\tilde{N}$ satisfies the following conditions:

(i) $d$ is $\rho\left(\pi_{1}(N)\right)$-invariant;

(ii) $d$ is a path semimetric;

(iii) $N$ has finite $d$-diameter;

(iv) any set of finite $d_{H}$-diameter in $\tilde{N}$ has finite $d$-diameter; and

(iv) any set of finite $d$-diameter in $\tilde{N}$ has finite $d_{H}$-diameter.

Given these properties the theorem is proved in [2] for the fibering case. It is immediate to generalize their proof to our situation.

We proceded to check properties (i) $-(\mathrm{v})$. Only properties (iii) $-(\mathrm{v})$ need checking. Let $x \in N$ in the spiral and $y=p_{1}(x)$. Since the $g$-flow segment between $x$ and $y$ is contained in the spiral, $d(x, y)=0$. On the core $d t$ is quasiconformal with hyperbolic length along $g$-flow lines and the transverse laminations $\pi\left(V_{+} \times \mathbf{R}\right) \cap C(N)$ and $\pi\left(V_{-} \times \mathbf{R}\right) \cap C(N)$ are compact sets. Therefore $N$ has finite $d$-diameter.

Let $U \subset \widetilde{N}$ be a set of finite hyperbolic diameter. Then it is contained in a union of finitely many translates of a fixed fundamental domain for $N$ in $\tilde{N}$, each of which has finite $d$-diameter, so (iv) follows.

The hard property to prove is $(\mathrm{v})$. Let $U$ be a set in $\widetilde{N}$ of $d$-diameter $e$. Since $d$ is $\rho\left(\pi_{1}(N)\right)$-invariant and $N$ has bounded $d$-diameter, we may assume there is $x \in U \cap(\widetilde{T} \times\{0\}) \cap C(\tilde{N})$. If $U$ does not have finite hyperbolic diameter there are $x_{n} \in U$ with $d_{H}\left(x_{n}, x\right) \rightarrow+\infty$ but $d\left(x_{n}, x\right)<e$. If $x_{n} \in$ spiral then $y_{n}=p_{1}\left(x_{n}\right)$ is in the core and $d\left(y_{n}, x_{n}\right)=0$ so $d\left(y_{n}, x\right)<e-$ no atoms need to be crossed. By Proposition 5.4 the hyperbolic distance between $y_{n}$ and $x_{n}$ is bounded and therefore $d_{H}\left(y_{n}, x\right) \rightarrow+\infty$. Let $y_{n}=\left(z_{n}, t_{n}\right)$, where $z_{n} \in \widetilde{T}$, $t_{n} \in \mathbf{R}$.

Claim. $\left|t_{n}\right|<e$. Let $\gamma_{n}^{\prime}$ be a path connecting $x$ and $y_{n}$ of $d$-length $<e$. Then $\gamma_{n}=p_{1}\left(\gamma_{n}^{\prime}\right) \subset C(\tilde{N})$ and $l\left(\gamma_{n}\right)<e$. Recall that in the core 
$h_{+}, h_{-}=1$ so

$$
e>\int_{\gamma_{n}} d s \geq \int_{\gamma_{n}}\left(h_{+} h_{-}\right)|d t|=\int_{\gamma_{n}}|d t| \geq\left|t_{n}\right| .
$$

Let $y_{n}^{\prime}=\left(z_{n}, 0\right)$ be the projection of $y_{n}$ to $\widetilde{T} \times\{0\}$ along $g$-flow lines. Since $t_{n}$ is bounded and hyperbolic length along flow lines is bounded by a multiple of $d t$-length, $y_{n}^{\prime}$ is at a bounded hyperbolic distance from $y_{n}$ and therefore $d_{H}\left(x, y_{n}^{\prime}\right) \rightarrow+\infty$. Since they are on the same $g$-flow lines, $d\left(y_{n}, y_{n}^{\prime}\right) \leq\left|t_{n}\right|$ is bounded by $e$, therefore $d\left(y_{n}^{\prime}, x\right)$ is bounded.

Project $\gamma_{n}$ to $\beta_{n} \subset \widetilde{T} \times\{0\}$ along $g$-flow lines. The fact that $|t|<$ $e$ uniformly in $\gamma_{n}$ implies $\beta_{n} \subset \widetilde{R}$ for all $n$, where $R$ is the surface $C \cup\left(\bigcup_{i=0}^{c=[e]+1}\left(A_{i} \cup B_{i}\right)\right)$. This is because a point in the core of $T$ can move at most one step into the ladder by $g$. Consider the basic stable and unstable lengths of $\gamma_{n}$ (not including the remaining $d s_{s}$ factors). These lengths are uniformly bounded. Since every point in $\gamma_{n}$ is moved at most $c$ units in the $t$ direction these lengths are perturbed by at most a factor of $\lambda^{c}$, so the final basic transverse measures of $\beta_{n}$ are bounded. Now $R$ is a compact hyperbolic surface with geodesic boundary and $\left(V_{+} \cup V_{-}\right) \cap R$ is a union of transverse geodesic laminations which bind $R$ and the basic transverse measures have full support. Proposition 5.7 shows that the hyperbolic metric and the transverse measure semimetric are quasicomparable in $\widetilde{R}$ and therefore the $d_{T}$-length of $\beta_{n}$ is bounded. As a result, so is the length induced from $\widetilde{T} \times\{0\} \subset \mathbf{H}^{3}$. This implies that the hyperbolic distance from $x$ to $y_{n}^{\prime}$ is bounded, a contradiction.

This completes the proof of the quasi-isometry theorem. q.e.d.

Notice that the remaining $d s_{s}$ part of the semimetric was not used in this last part of the quasicomparability result because the union of the laminations $V_{+}$, and $V_{-}$binds a bigger subsurface of $T$ which is still compact. The essential use of the $d s_{s}$ part is in the fact that the push off map is distance decreasing as a consequence the analysis can be restricted to the core which is compact. Without the push off property it would be easy to produce counterexamples to quasicomparability by paths starting in the core going deep in the spiral, winding around a lot near $\partial N$ producing no $d$-length, and then returning to the core.

It is in fact true that foliation produces a semimetric in the original manifold $M$ which is quasi-isometric to the hyperbolic metric in the universal cover. In order to show this we consider the original components $Q$ of $M-\mathscr{C}$ in $Q$ of $M-\mathscr{C}$ instead of the enlarged ones $(N)$. Obviously there is a semimetric $d_{1}$ in $Q^{\prime \prime}$ naturally associated to the semimetric $d$ 
in $N$ and it satisfies the quasicomparability property in $\widetilde{Q}$.

Introduce the semimetric $d_{1}$ on the finitely many components of this set. Given a compact leaf $S$ let $Q$ be a component of $M-\mathscr{C}$ on the negative side of $S$. Put an atomic measure across $S$ of atom $\mu_{S}<1 / 2$. In $Q$ the transverse measure to $\pi\left(V_{+} \times \mathbf{R}\right)$ induces a generalized transverse measure to $V_{+} \downarrow S_{\varepsilon}$ in $S_{\varepsilon}$. The $g$-flow then induces a generalized transverse measure to $V_{+} \downarrow S$, the geodesic lamination induced in $S$. Add this measure to $S$ and do the same for the positive side. Also add the juncture measures.

Theorem 5.9. Under the conditions of the main theorem the semimetrics $d_{1}$ and $d_{H}$ are quasi-isometric in $\widetilde{M}=\mathbf{H}^{3}$.

Proof. Apply the same strategy as in Theorem 5.8. Everything goes through immediately except for (iii) and (v) of the claim. Let $\mathscr{\mathscr { C }}=$ $\pi^{-1}(\mathscr{C})$. To show (iii) move boundedly in $\mathscr{C}$ to a point not in the projection of the invariant laminations or juncture annuli. Then use the well-defined projection of the $g$-flow to move out of $\tilde{\mathscr{C}}$. To show (v) let $p, q \in \widetilde{M}$ with $d_{1}(p, q)<e$. By moving them a little assume $p, q \notin \widetilde{\mathscr{C}}$ (by assuming $d_{1}(p, q)<e+k=e^{\prime}$, where $k$ is a fixed constant). Take a path $\gamma$ connecting them with $l_{1}(\gamma)<e^{\prime}$. Since in the spirals $l_{1}$-length is $\leq 2 d s_{s}$-length we can change the path so that $l_{1}(\gamma)<2 e^{\prime}$ but $\gamma$ is transverse to $\widetilde{\mathscr{C}}$. Since $l_{1}(\gamma)<2 e^{\prime}$ it intersects $\tilde{\mathscr{C}}$ in a bounded number of points $p_{1}, \cdots, p_{n}$. Let $p_{0}=p$ and $p_{n+1}=q$. The quasicomparability theorem then shows that $d_{H}\left(p_{i}, p_{i+1}\right)$ is bounded and therefore so is $d_{H}(p, q)$. This completes the proof.

\section{Flat walls}

In this section we study 2-dimensional submanifolds of $\widetilde{N}$ (or $N$ ) which will be the key ingredient in the analysis of the asymptotic behavior of the leaves of the foliation. They will be quasigeodesic, a property more visible in the semimetric $d$ which will then be interpreted in the hyperbolic metric using the quasi-isometry relation between them.

A set $D$ in a space $E$ with a path semimetric $e$ is flat if for any two points $p, q \in D$ and any path $\gamma^{\prime} \subset E$ connecting them there is also a path $\gamma \subset D$ connecting them with $l_{e}(\gamma) \leq l_{e}\left(\gamma^{\prime}\right)$. This is the natural generalization of totally geodesic in Riemannian manifolds. But in general there is no uniqueness of minimal paths because $e$ is only a semimetric.

Let $L$ be a leaf of $\Gamma^{s} \cup \Gamma^{u}$. We identify $L$ to a lift $L$ in $\widetilde{T}$ and also to its image in $\widetilde{T} \times\{0\}$. The saturation of $L$ in $\widetilde{N}$ by the $g$-flow $(L \times \mathbf{R})$ is 
called a wall. Our aim is to prove that a wall is flat in the $d$ semimetric. This would easily work if $d$ only had the pseudo-Anosov factors, but the existence of the $d s_{s}$ factors complicate the analysis. We will show that the part of the wall in the core is flat, which will be enough for our purposes.

The description of a typical wall is as follows: Let $L \subset \Gamma^{\mathcal{s}}$. The induced metric (from $\mathbf{H}^{3}$ ) in $L \times\{0\}$ identifies it to the real numbers. Parametrize $L \times \mathbf{R}$ by $(w, t)$, where $(\cdot, t)$ is in a $g$-flow line and $w$ is given by the above identification. Consider $t^{\prime}=\lambda^{t}$, which identifies $L \times \mathbf{R}$ with the upper half plane in $\mathbf{R}^{2}$. Again vertical lines are flow lines, and horizontal ones are components of wall $\cap \widetilde{F}$. Locally $\Gamma^{u}$ intersects $L$ in an isolated point or a Cantor set. This intersection is invariant under the $g$-flow. Let $c$ be a complementary region of $\Gamma^{u}$ in $L$ with $c \cap \mathscr{U}_{-}=\varnothing$ (here $L$ is a subset of $T$ ). In $T$, any point $w$ in $c$ satisfies: $g^{i}(w) \notin$ negative ladder $\forall i \in \mathbf{Z}$. Since $c \subset \Gamma^{\mathcal{S}}, g^{i}(w) \notin$ positive ladder either, therefore $c \times \mathbf{R} \subset C(\tilde{N})$. If $c^{\prime}$ is a complementary region with $c^{\prime} \cap \mathscr{U}_{-} \neq \varnothing$ then $c^{\prime} \subset \mathscr{U}_{-}$(see [5]) and for any $w \in c^{\prime}, \exists i<0$ with $g^{i}(w) \in$ ladder, so the flow line through $(w, 0)$ eventually enters in the spiral in the negative direction and it will limit on $\partial_{-} \tilde{N}$. The boundary between the core and spiral regions in $c^{\prime} \times \mathbf{R}$ is a curve $\eta$ contained in $\partial_{-} \tilde{N} \times\{\varepsilon\}$. Recall that this curve is tangent to the foliation outside a neighborhood of the juncture, which accounts for the horizontal pieces of the curve. The thickness of the spiral in the hyperbolic metric is bounded by the maximum length of a $g$-flow segment from $\partial_{-} N \times\{\varepsilon\}$ to $\partial_{-} N$. An infinite complementary interval $e$ occurs when $L$ has an escaping ray. Except for a finite length, $e$ is entirely contained in the ladder and the same holds for $g^{i}(e), \forall i \in \mathbf{Z}$. Notice that the wall is not a complete submanifold because it has boundary in $\partial \widetilde{N}$ corresponding to segments in $L \cap \mathscr{U}_{-}$.

The upper half plane model of $L \times \mathbf{R}$ is roughly reminiscent of the same model of $\mathbf{H}^{2}$. To see the suppose for a moment there is no $d s_{s}$ factor (or $h_{+}, h_{-}$) in the semimetric and collapse the $0 d$-distant points to get a metric space (usually complete, unless there is an escaping ray in $L$ ). Then the metric is $d s=\left(|d y|+\left|d t^{\prime}\right| / \log \lambda\right) / t^{\prime}$ which is quasiconformal to the hyperbolic metric $\left(d \bar{s}^{2}=\left(d y^{2}+d t^{\prime 2}\right) / d t^{2}\right)$. Up to a bounded distortion, minimal arcs would behave as geodesics in $\mathbf{H}^{2}$. The $d s_{s}$ part of $d$ distorts this analogy to a certain extent, but not significantly because the spiral has bounded thickness in the flow direction.

Our objective is to produce a distance decreasing retraction from $\tilde{N}$ to $L \times \mathbf{R}$. Suppose again for a moment that there is no $d s_{s}$ factor and that $h_{+}, h_{-}=0$. Define the retraction $r^{\prime}: \widetilde{T} \rightarrow L$ as follows: if $L^{\prime} \in V_{+}$ 


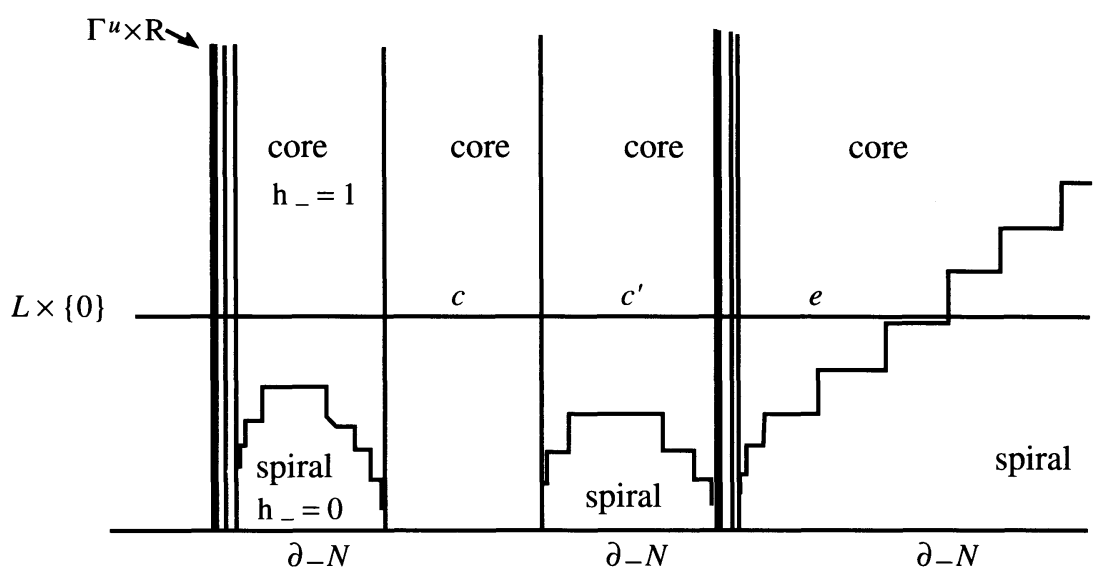

Figure 6. A TYPICAL WALL IN $\tilde{N}$.

intersects $L$ project $L^{\prime}$ to $L \cap L^{\prime}$. Project a complementary component $D$ of the union of these $L^{\prime}$ continuously to $D \cap L$ so as to be continuous, to agree with the projection defined on the boundary, and to fix the intersection with $L$. Extend this to $\tilde{N}$ by $r(w, t)=\left(r^{\prime}(w), t\right)$. This retraction would preserve the $d t$ factor, eliminate the $d x$ factor (no measure across $V_{-} \times \mathbf{R}$ since image is contained in this set), and more importantly would preserve or decrease the $d y$ factor. This last fact occurs because the image will only be transverse to $V_{+} \times \mathbf{R}$ in $L \times \mathbf{R}$, when the domain itself is and since $t$ is preserved the corresponding $d y$ factor does not increase. So the retraction would be distance decreasing. This is the basic construction, which will have to be adjusted to take into account the $d s_{s}$ factor and that $h_{+}, h_{-} \neq 0$.

First project $\tilde{N}$ to $C(\tilde{N})$ using the push of map $p_{1}$, which is distance decreasing. We define a projection $p_{2}: \widetilde{N} \rightarrow L \times \mathbf{R}$ which is distance decreasing in the core. The projection described above presents two potential problems:

(i) Points in the spiral (where $h_{+} h_{-}|d t|=0$ ) are projected to the core (where $h_{+} h_{-}|d t|=|d t|$ ) introducing $d t$-length. This is why the distance decreasing property of the projection works only in the core. There is no good way to make this hold everywhere while still preserving the height $t$.

(ii) Core points (where $\left.\left(1-h_{+} h_{-}\right) d s_{s}=0\right)$ are projected to spiral parts (where $\left(1-h_{+} h_{-}\right) d s_{s}=d s_{s} \neq 0$ ) and create $d s_{s}$-length. 
The solution to problem (ii) is as follows: Let $J=\left(\bigcup L^{\prime}\right)$, where the union is on $L^{\prime} \in \Gamma^{u}$ so that $L^{\prime} \cap L \neq \varnothing$. Project $J \times \mathbf{R} \rightarrow L \times \mathbf{R}$ as described above. For any $l \in W_{+}$with $l^{\prime} \cap L \neq \varnothing$ project $l^{\prime} \times \mathbf{R} \rightarrow$ $\left(l^{\prime} \cap L\right) \times \mathbf{R}$ as above, unless $l^{\prime} \cap L$ is in an escaping ray of $L$. Let $c$ be a component of $L-\Gamma^{u}$ and $D_{c}$ the complementary region of $J$ in $\widetilde{T}$ satisfying $c=D_{c} \cap L$. If $c \cap \mathscr{U}_{-}=\varnothing$ then $(c \times \mathbf{R}) \subset$ core and no $d s_{s}$ measure is created by any projection to $c \times \mathbf{R}$, so project $D_{c} \times \mathbf{R}$ continuously to this set preserving $t$ and so that it is coherent with the previously defined projection on $\left(V_{+} \cap D_{c}\right) \times \mathbf{R}$. If $c$ is an escaping ray with boundary point $e \in \Gamma^{u}$ define the projection in $c \times \mathbf{R}$ by $(w, t) \rightarrow(e, t)$.

Finally let $c \subset \mathscr{U}_{-}$be a component of $L-\Gamma^{u}$ which is not escaping. Then the elements of $d s$ in $c \times \mathbf{R}$ are the following:

(1) the vertical factor $|d t|$ in the core,

(2) $\lambda^{-t} d y$ measure across $W_{+} \times \mathbf{R}$ in the core which is kept fixed in the spiral, and

(3) measure induced from $|d t|$ in the negative spiral.

We define a retraction $p_{2}: D_{c} \times \mathbf{R} \rightarrow c \times \mathbf{R}$ which has the form $p_{2}(z, t)=$ $(p(z), t)$, where $p: D_{c} \rightarrow c$ is a retraction.

Let $\partial c=a \cup b$ both in $\Gamma^{u}$. Recall $\eta$ is the boundary of the core part in $c \times \mathbf{R}$. The intersections of negative juncture barriers with $c \times \mathbf{R}$ are quadrilaterals with one bent side. The upper boundary is contained in $\eta$ and is not in a leaf of the foliation, the lower boundary $\subset \partial_{-} \widetilde{N}$, and the laterals are $g$-flow segments (see Figure $7(a)$ ). There are countably many of these corresponding to each "step" in $\eta$. Let $\alpha_{i}, \beta_{i}$ be these intersections of juncture barriers with $c \times \mathbf{R}$ (one sequence for each side of $\eta$ ). The $g$-flow saturation of $\alpha_{i}\left(\beta_{i}\right)$ intersects $L \times\{0\}$ in $a_{i}\left(b_{i}\right)$, which are segments. The only limit point of $a_{i}$ is $a$ and similarly for $b_{i}$ (see Figure 7(a)). If there were any other limit points then different junctures would accumulate in $\widetilde{N}$, a contradiction.

Recall that the juncture barriers are the only places where segments contained in a leaf of $\mathscr{F}$ have $d$-length coming from $|d t|$. Consider the juncture barrier $H_{i}$ which intersects $c \times \mathbf{R}$ in $\alpha_{i}$. The intersection of the $g$-flow saturation of $H_{i}$ with $\widetilde{T} \times\{0\}$ is denoted by $\alpha_{i}^{*}$. Then $\partial \alpha_{i}^{*}=\phi_{1} \cup \phi_{2}$, both projecting to $N$ as closed curves, where $\phi_{1}$ is geodesic and corresponds to a $g$-flow translate of the negative juncture (that is, $\phi_{1} \in$ $W_{+}$) and $\phi_{2}$ is a $g$-flow translate of $\delta^{\prime}$, the other boundary component of the juncture annulus. Notice that if $r \in W_{+}$then $r \times \mathbf{R}$ can only intersect a juncture barrier in one of its lateral boundaries.

We already projected $\phi_{1} \times \mathbf{R}$ to $\left(\phi_{1} \cap a_{i}\right) \times \mathbf{R}$ by $(x, t) \rightarrow$ $\left(\left(\phi_{1} \cap a_{i}\right), t\right)$ when considering $W_{+} \cap D_{c}$. Do the same for $\phi_{2} \times \mathbf{R}$. Project 


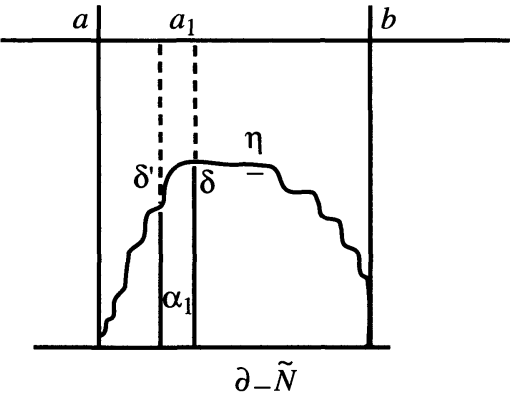

(a)

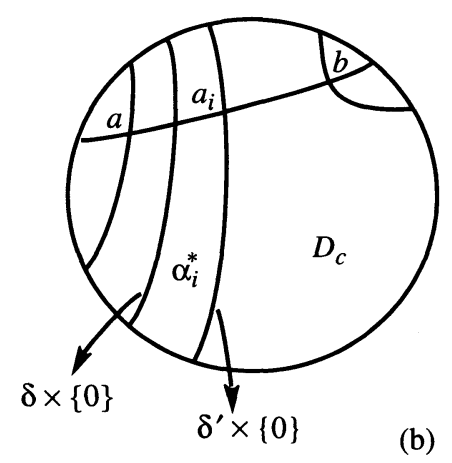

(b)

Figure 7. The WALl AND The SPIRAL.

$H_{i}$ continuously to $\alpha_{i}$ preserving the juncture measure and $t$, and so as to agree with projection already defined in $\partial \alpha_{i}^{*} \times \mathbf{R}$. The upper boundary of $H_{i}$ is an infinite band which should be projected to $\eta \cap H_{i}$. Finally project $\left(\alpha_{i}^{*} \times \mathbf{R}\right)-H_{i}$ continuously to $\left(a_{i} \times \mathbf{R}\right)-\alpha_{i}$ so as to preserve $t$ and agree with projection already defined on the boundary. Do this for all $\alpha_{i}$ and $\beta_{i}$.

Let now $E$ be a component of $D_{c}-\left(\bigcup_{i \in N}\left(\alpha_{i}^{*} \cup \beta_{i}^{*}\right)\right)$. Project $E \times \mathbf{R}$ to $(E \cap c) \times \mathbf{R}$ preserving $t$ and coherently with the projection already defined on the boundary. Do this for all such complementary regions $c$ producing $p_{2}: \widetilde{N} \rightarrow L \times \mathbf{R}$.

Proposition 6.1. The projection $p_{2}: \widetilde{N} \rightarrow L \times \mathbf{R}$ is distance decreasing in the core.

Proof. This function is continuous by construction. By definition $p_{2}$ preserves $t$, therefore it can only decrease $|d t|$ when restricted to the core. The transverse measure to $V_{+} \times \mathbf{R}$ is also preserved or decreased because leaves of $V_{+}$intersecting $L$ are projected to the intersection and complementary regions are taken to complementary regions and $t$ is preserved. This is even true for $W_{+} \times \mathbf{R}$, at lest in the core. Notice the stabilization of the measure in the spiral does not destroy this property because $g^{n}\left(\partial_{-} C\right)$ will intersect the negative ladder if and only if it is contained in it. Therefore the distortion in the transverse measure is the same for all points at a given height $t$.

The only problem may occur when part of an arc is projected transversally to one of the corresponding $\alpha_{i}$ or $\beta_{i}$. By construction this only 
happens when the original arc was already transverse to the corresponding juncture barrier and its measure is the same as that of the projection. Therefore now new juncture measure is created. This completes the proof of the proposition. q.e.d.

Notice that, if $L$ has no escaping rays, $p_{2}$ fixes $L \times \mathbf{R}$. Otherwise $p_{2}$ fixes $(L-e) \times \mathbf{R}$, where $e$ is the union of escaping rays. Sometimes there can be two such rays in a leaf, as shown in [4]. If $L$ does not contain an escaping ray let $C_{L}=(L \times \mathbf{R}) \cap C(\tilde{N})$. Otherwise let $C_{L}=$ $((L-e) \times \mathbf{R}) \cap C(\widetilde{N})$, where $e$ is the union of the escaping rays of $L$.

Theorem 6.2. For any $L \in \Gamma^{\top} \cup \Gamma^{u}, C_{L}$ is flat in the semimetric $d$.

Proof. Let $\mathrm{pr}=p_{1} p_{2} p_{1}$. By Propositions 5.2 and 6.1, $\mathrm{pr}$ is distance decreasing. Furthermore, $p_{1}$ and $p_{2}$ fix $C_{L}$. As $d$ is a path semimetric the result follows.

Lemma 6.3. Given $L \in \Gamma^{u} \cup \Gamma^{\mathcal{S}}$ and $p, q \in C_{L}$ there is a path $\gamma \subset C_{L}$ connecting them with $l(\gamma)=d(p, q)$.

Proof. This is not immediate because $d$ is not even a metric. In fact the proof is constructive and these paths will be used later. Assume $L \in$ $\Gamma$. To find candidates for minimal paths we may assume $\gamma \subset C_{L}$ by the previous theorem, therefore only $|d t|$ and $\lambda^{-t} d y$ are present.

Parametrize a path $\gamma \subset C_{L}$ connecting $p, q$ by $\gamma(s)=(w(s), t(s))$, $s \in[0,1]$. Let $e=\max _{s \in[0,1]} t(s)$. Let $\gamma^{\prime}$ be a path starting at $\gamma(0)$, moving vertically (that is, along a $g$-flow line) to $(w(0), e)$, then horizontally (along the foliation) to $(w(1), e)$, and finally vertically down to $(w(1), t(1))=q$ (see Figure 8). The path $\gamma^{\prime}$ is always above $\gamma$ in the

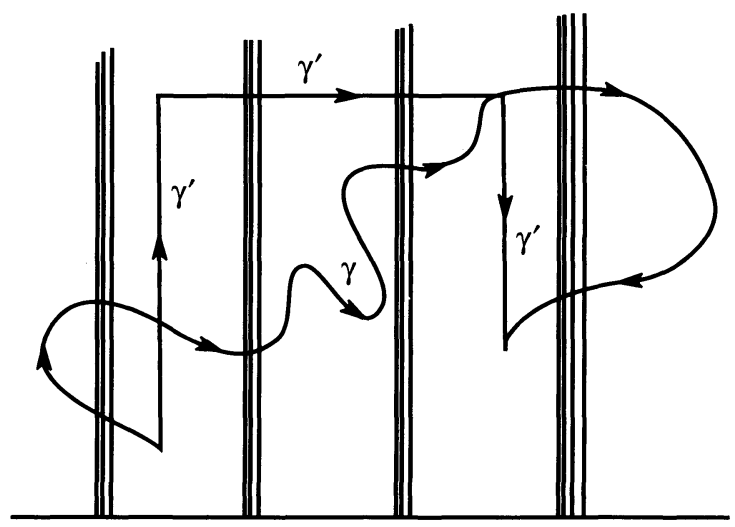

Figure 8. How to DeCreAse Lengths OF PATHS. 
sense that for every $\left(w, t^{\prime}\right) \in \gamma^{\prime}$ there is $(w, t) \in \gamma$ with $t \leq t^{\prime}$. This implies that $\gamma^{\prime} \subset C_{L}$ and furthermore, by construction $\int_{\gamma^{\prime}}|d t| \leq \int_{\gamma}|d t|$. Since the $d y$ factor decreases exponentially under the positive direction of the $g$-flow the $d y$ part of the length of $\gamma^{\prime}$ is bounded by that of $\gamma$. Consequently $l\left(\gamma^{\prime}\right) \leq l(\gamma)$.

Let $c^{\prime}=\min \{t \mid(w(0), w(1)) \times\{t\} \subset \operatorname{core}(\tilde{N})\}$. For any $c \geq c^{\prime}$ there is a path $\gamma_{c} \subset C_{L}$ connecting $p, q$ of the form: vertically from $(w(0), t(0))$ to $(w(0), c)$, horizontally from $(\omega(0), c)$ to $(\omega(1), c)$ and finally vertically to $(w(1), t(1))$. The length $l\left(\gamma_{c}\right)$ is a continuous function of $c$. This function is a convex function and therefore assumes a unique minimum at some $c \geq c^{\prime}$. Then $\gamma(p, q)=\gamma_{c}$ is a path satisfying the requirements of the lemma. q.e.d.

There is no uniqueness of minimal length paths, because for instance $\gamma$ may move more horizontally, without crossing $\Gamma^{u} \times \mathbf{R}$, which does not increase its $d$-length. The path $\gamma(p, q)$ described above is called a good minimal path. The notation $\gamma(p, q)$ is reserved for these paths.

It is an interesting fact that the metric $d s=\left(|d y|+\left|d t^{\prime}\right| / \log \lambda\right) / d t^{\prime}$ in the upper half plane produces minimal paths as described above and all but a bounded length is traversed vertically. Notice that these paths are always a bounded distance from the hyperbolic geodesic ones.

Lemma 6.4. There are $\nu, \mu>0$ so that for any $L \in \Gamma^{u} \cup \Gamma^{s}$ and any two points $p, q \in C_{L}, l_{H}(\gamma(p, q)) \leq \nu d(p, q)+\mu$.

Proof. Let $\gamma_{1}$ be the horizontal part of the path $\gamma(p, q)$, and $\gamma_{2}$ its vertical part. In the core, $|d t|$ is quasiconformal with hyperbolic length along $g$-flow lines, so there is $\nu>0$ with $l_{H}\left(\gamma_{2}\right) \leq \nu l\left(\gamma_{2}\right)$. This $\nu$ is independent of $p, q$, and even $L$.

We claim that $l_{H}\left(\gamma_{1}\right)$ is bounded by some $\mu>0$. Otherwise there is $\gamma_{1}^{i}$ with $l_{H}\left(\gamma_{1}^{i}\right)>i$. But $\gamma_{1}^{i} \subset \widetilde{T} \times\{t(i)\}$. By compactness of the core we can assume $t(i) \rightarrow t_{0}$ and that $\gamma_{1}^{i} \rightarrow \gamma_{0}$. Then $\gamma_{0}$ is infinite, but $\gamma_{0} \times\left\{t_{0}\right\} \subset\left(\Gamma^{\mathcal{S}} \times \mathbf{R}\right) \cap C(\tilde{N})$ and there are no infinite horizontal arcs of $\Gamma^{s} \times \mathbf{R}$ in the core because projection to $\widetilde{T} \times\left\{\left[t_{0}\right]+1\right\}$ would produce an infinite arc of $\Gamma^{s} \subset C(T)$. But $\Gamma^{s}$ intersects the core in a finite union of bands of isotopic finite arcs, so this is impossible. Then

$$
l_{H}(\gamma(p, q))=l_{H}\left(\gamma_{1}\right)+l_{H}\left(\gamma_{2}\right) \leq \nu l\left(\gamma_{2}\right)+\mu \leq \nu d(p, q)+\mu
$$

Proposition 6.5. There are $r, u>0$ so that: if $L \in \Gamma^{s} \cup \Gamma^{u}$, then any good minimal path in $C_{L}$ is as $(r, u)$ quasigeodesic curve in the hyperbolic metric for $\tilde{N}$. 
Proof. Given any two points $p, q \in C_{L}$ they are in the core of $\tilde{N}$, therefore in its convex hull. The hyperbolic geodesic connecting them is contained in $\widetilde{N}$ and then obviously it is the minimal path in the hyperbolic metric on $\tilde{N}$. This is the reason why in $\S 4$ we modified the original manifold $Q$ to obtain $N$ containing its convex hull and whose core is contained in the convex hull. Let $k, K$ be constants satisfying the quasicomparability inequalities between $d_{H}$ and $d$. Take $\nu$ and $\mu$ given by Lemma 6.4. Let $u=K \nu+\mu$. Let $\gamma$ be a good minimal path in $C_{L}$ and $\alpha$ any subpath with hyperbolic length $>u$ and endpoints $p, q$. Notice that any subpath of a a good minimal path is itself also a path of the same type, so $l(\alpha)=d(p, q)$. If $d(p, q) \leq K$, then since $\alpha$ is a $d$-minimal path, Lemma 6.4 implies that $l_{H}(\alpha) \leq K \nu+\mu=u$, a contradiction. Furthermore the same lemma implies

$$
l_{H}(\alpha) \leq b^{\prime} d(p, q),
$$

where $b^{\prime}=\nu+\mu / K$ (because $\left.K<d(p, q)\right)$.

By quasicomparability $d(p, q) \leq 2 k d_{H}(p, q)$. As a result

$$
l_{H}(\alpha) \leq k b^{\prime} d_{H}(p, q) \text {. }
$$

Take $r=b^{\prime} k$. Note $r$ and $u$ are independent of $p, q$, and $L$. q.e.d.

Thurston [17] showed that an $(r, u)$ quasigeodesic curve in $\mathbf{H}^{3}$ is at a bounded distance from the hyperbolic geodesic arc connecting the endpoints. This shows:

Theorem 6.6. $\quad C_{L} \subset \mathbf{H}^{3}$, where $L \in \Gamma^{u} \cup \Gamma^{s}$, is uniformly quasigeodesic in the hyperbolic metric.

Remark. Consider the annuli $G_{j}$ which are the $g$-flow saturations in $N$ of the junctures. We conjecture that lifts of these to $\tilde{N}$ are also uniformly quasigeodesic. If this is true it simplifies considerably the proof of the continuous extension of $i$. Unfortunately the corresponding projection $p_{2}$ is not distance decreasing in the core. The problem is that measure across $V_{+} \times \mathbf{R}, V_{-} \times \mathbf{R}$ may increase, even multiplied by an enormous factor, under the projection $p_{2}$ to $L \times \mathbf{R}$, with $L \in W_{-} \cup W_{+}$.

Lemma 6.7. Any point in $L \times \mathbf{R}$ is at a bounded $d$ (and $d_{H}$ ) distance from $C_{L}$ in $L \times \mathbf{R}$.

Proof. If $L$ does not contain an escaping ray, then any point in $L \times \mathbf{R}$ is either in $C_{L}$ or in the spiral. The spiral has zero $d$-thickness in the $g$-flow direction. By Proposition 5.4 the hyperbolic length of these flow segments in the spiral is bounded so the result holds for these types of leaves. If $e$ is the union of the escaping rays, the same argument will work for $(L-e) \times \mathbf{R}$. Let $\beta=\partial(e \times R)$ be a flow line contained in 
$C_{L}$. Any point $(x, t)$ in $C(\tilde{N}) \cap(e \times \mathbf{R})$ is at a bounded hyperbolic and therefore also $d$-distance from $\beta$ by a horizontal segment. Any other point is in the spiral and the argument above applies.

Corollary 6.8. $L \times \mathbf{R}$, where $L \in \Gamma^{u} \cup \Gamma^{s}$, is uniformly quasigeodesic in $\mathbf{H}^{3}$.

Proof. Let $p, q \in L \times \mathbf{R}$. Let $\nu>0$ be the hyperbolic bound given by the previous lemma. There are $p^{\prime}, q^{\prime} \in C_{L}$ so that $d_{H}\left(p^{\prime}, p\right)<\nu$ and similarly for $q, q^{\prime}$. Let $\gamma, \beta$ be the hyperbolic geodesic arcs connecting $p, q$ and $p^{\prime}, q^{\prime}$ respectively. Since the endpoints of $\beta$ are at most $\nu$ distant from those of $\gamma$ it follows from hyperbolic geometry that $\beta$ is entirely contained in the $\nu$-neighborhood of $\gamma$. The previous theorem implies that every point of $\beta$ is at most $\tau+\nu$ distant from $C_{L}$, where $\tau$ is a constant.

\section{Existence and continuity}

We now address the main theorem: there is a continuous extension of $\varphi_{\widetilde{T}}: \mathbf{H}^{2} \rightarrow \mathbf{H}^{3}$ to $\mathbf{H}^{2} \cup S_{\infty}^{1}$. Both the existence and the continuity will use in an essential manner the quasigeodesic property of the walls. Recall that under the homeomorphism $\widetilde{N} \cong \widetilde{T} \times \mathbf{R}, \varphi_{\widetilde{T}}(\widetilde{T})$ is associated to $\widetilde{T} \times\{0\}$.

Lemma 7.1. Let $r$ be an infinite ray in $T-\left(\Gamma^{s} \cup \Gamma^{u}\right)$. Except for a finite length, $r$ is contained in one of the ladders.

Proof. Suppose $r$ keeps returning to the core. If it self-intersects it defines a homotopically nontrivial closed curve which is disjoint from the laminations, a contradiction to irreducibility. In the other case, $r$ limits on $w \in C$. If $\omega \notin \Gamma^{s} \cup \Gamma^{u}$ so does a small neighborhood $U$ of $w$ and we can connect a big enough subarc of $r$ with endpoints in $U$ to again produce a closed curve as above.

Otherwise $w \in \Gamma^{u}$, say, and let $l$ be the leaf of $\Gamma^{u}$ through $w$. Then $l$ intersects $\Gamma^{s}$ in at least a leaf $l^{\prime}$. Since $r$ limits on $w$ but $r$ does not intersect $l$, the tangent to $r$ also limits on the tangent direction of $l$ at $w$. This implies that $r$ will also intersect $l^{\prime}$, a contradiction.

Theorem 7.2. There is a natural extension $\bar{\varphi}_{\widetilde{T}}$ of $\varphi_{\widetilde{T}}: \widetilde{T}=\mathbf{H}^{2} \rightarrow \mathbf{H}^{3}$ to $S_{\infty}^{1}$ with image in $S_{\infty}^{2}$.

Proof. Recall that the hyperbolic metric in $\widetilde{T}$ is induced from one in $T$ and $f$ is an isometry in the ladders. The map $g$ on the other hand is not an isometry in the ladders but it preserves the invariant laminations. Let $p \in S_{\infty}^{1}$ be an ideal point of $\widetilde{T}$ and $r$ a geodesic ray in $\widetilde{T}$ with this ideal point. We are going to show that $\varphi_{\widetilde{T}}(r)$ has a well defined limit 
point in $S_{\infty}^{2}$. This is independent of the particular ray chosen, for any other ray is asymptotic to $r$, and since the hyperbolic structure in $\varphi_{\widetilde{T}}(\widetilde{T})$ is quasiconformal to the induced metric in $\varphi_{\widetilde{T}}(\widetilde{T}) \subset \mathbf{H}^{3}$ their images will be asymptotic in $\mathbf{H}^{3}$. There are two cases:

(I) Type 1. The ray $r$ eventually stops intersecting $\Gamma^{u} \cup \Gamma^{s}$. Assume then that $r$ does not intersect either of these laminations by elminating that $r$ is contained in the ladder except perhaps for a finite length subsegment, so assume it is entirely contained in the ladder. Projection along the original flow ( $f$-flow) to the component of $\partial \widetilde{N}$ associated to the part of the spiral containing $\varphi_{\widetilde{T}}(r)$ produces a ray which is a finite hyperbolic distance (bounded by the thickness of the ladder under the $f$-flow) from the original ray. In the intrinsic hyperbolic metric of this component the projection is a geodesic (because $f$ is an isometry in the ladders) and as $\partial N$ is quasi-Fuchsian this ray will have as image a quasigeodesic in $\mathbf{H}^{3}$ which has a well-defined limit point. This is $\bar{\varphi}_{\widetilde{T}}(p)$.

(II) Type 2. The ray $r$ keeps intersecting the laminations. This is the hard case. If the intersection is not transversal then $r \subset \Gamma^{u} \cup \Gamma^{s}$, say $\Gamma^{u}$. If $r$ eventually stops intersecting $\Gamma^{s}$ transversally then $r$ is eventually contained in an escaping ray of $\Gamma^{u}$, which is contained in the ladder and we reduce to case I. Else $r$ keeps intersecting $\Gamma^{s}$ transversally. Assume then $r$ keeps intersecting $\Gamma^{s}$ (say) transversally.

Let $z$ be the starting point of $r$. By taking another asymptotic ray if necessary we may assume $z$ is in the core. Let $L_{n} \in \Gamma^{S}$ so that $L_{n} \rightarrow \infty$ in $\widetilde{T}$ and $L_{n}$ intersects $r$ transversally. Let $r_{n}$ be the subray of $r$ between $L_{n}$ and $p$. Let $y=\varphi_{\widetilde{T}}(z)$. In $\widetilde{T}, \lim _{n \rightarrow+\infty} d_{T}\left(z, L_{n}\right)=+\infty$.

Lemma 7.3. $\lim _{n \rightarrow+\infty} d_{H}\left(y, L_{n} \times \mathbf{R}\right)=+\infty$.

Proof. Otherwise up to subsequence there is $\mu>0$ and $y_{n} \in L_{n} \times \mathbf{R}$, with $d_{H}\left(y_{n}, y\right)<\mu$. If $y_{n} \in$ spiral it flows back to $C(\tilde{N})$ under a bounded hyperbolic length $g$-flow segment, so assume $y_{n} \in C(\widetilde{N})$. By Theorem 5.8, $d\left(y_{n}, y\right)<\nu$ for some fixed $\nu>0$. Choose $\gamma_{n} \subset C(\tilde{N})$ connecting $y$ and $y_{n}$ and $l\left(\gamma_{n}\right)<\nu$. As in the proof of the same theorem $t$ is uniformly bounded in $\gamma_{n}$. Therefore the basic measure length of the projection $\gamma_{n}^{\prime}$ of $\gamma_{n}$ to $\widetilde{T} \times\{0\}$ along the $g$-flow is also bounded. Notice $y \in \tilde{Y} \times\{0\}$. By Proposition 5.7 the endpoints of $\gamma_{n}^{\prime}$ are a bounded distance apart in the intrinsic hyperbolic metric of $\widetilde{T} \times\{0\}$. But one of them is $y$ and the other is in $L_{n} \times\{0\}$ so $d_{T}\left(L_{n}, z\right) \nrightarrow+\infty$, a contradiction. q.e.d.

Consider the unit disk model for $\mathbf{H}^{3}$ with classical compactification $B R=\mathbf{H}^{3} \cup S_{\infty}^{2}$, the closed unit ball in $\mathbf{R}^{3}$. The visual distance in $B R$ is 


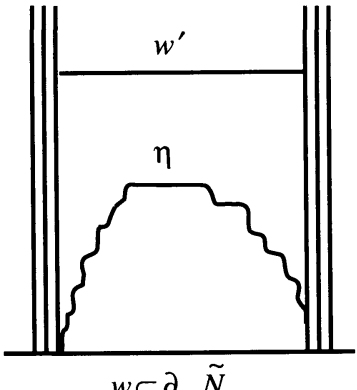

(b)

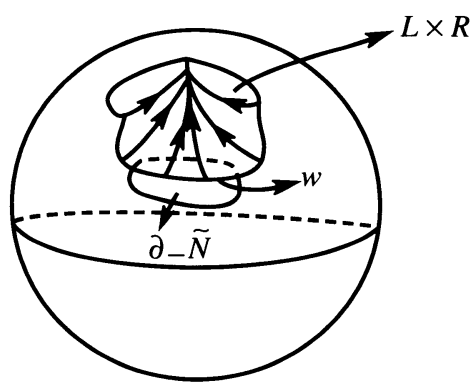

(a)

Figure 9. Boundary OF WALLS IN $\mathbf{H}^{3}$.

the induced metric from the inclusion in $\mathbf{R}^{3}$ with the euclidean metric. The same is done for the unit disk model of $\mathbf{H}^{2}$. The term visual will always refer to this model. Let $v_{d}$ be the visual diameter of a set and $v$ the visual distance.

Notice $L_{n} \times \mathbf{R}$ has infinite hyperbolic diameter. As the walls are going to infinity in $\mathbf{H}^{3}$ and they are uniformly quasigeodesic they satisfy $v_{d}\left(L_{n} \times \mathbf{R}\right) \rightarrow 0$.

We are almost done except for the fact that the walls do not separate $\mathbf{H}^{3}$. This happens because $\tilde{N}$ is not a complete submanifold of $\mathbf{H}^{3}$. $L_{n} \times \mathbf{R}$ has boundary in copies of lifts of $\partial N$, corresponding to flow lines which hit $\partial_{-} \tilde{N}$ in backward time, as in Figure 9.

To fix this adjoin half disks in $\partial_{-} \tilde{N}$ to $L_{n} \times \mathbf{R}$. Let $w^{\prime} \subset \mathscr{U}_{-}$be a complementary segment of $\Gamma^{u}$ in $L_{n}$. Then $w$, the projection of $w^{\prime}$ to $\partial_{-} \tilde{N}$ (using the $g$-flow), defines a curve which, by Proposition 5.5 , is a geodesic $w^{*}$ in the intrinsic hyperbolic metric of this set. Therefore it is a quasigeodesic in $\mathbf{H}^{3}$. Be aware that $w^{\prime}$ is not at a finite hyperbolic distance from $w$ because the distance goes to infinity near the boundary of $w^{\prime}$.

Let $\widetilde{S}_{w}$ be the component of $\partial_{-} \tilde{N}$ which contains $w$. Then $w$ separates it into two disk components $W_{w}$ and $U_{w}$. The curve $w$ has small visual diameter because it is on the boundary of the wall, which has this property. The next result shows that small visual curves in quasi-disks bound visually small half disks.

Lemma 7.4. At least one of the components $W_{w}, U_{w}$ has small visual diameter.

Proof. Else there is $\mu>0$ and a sequence $w_{n}^{\prime} \in L_{n}$ of complementary segments so that $v_{d}\left(w_{n}\right) \rightarrow 0$ but $v_{d}\left(W_{w_{n}}\right), v_{d}\left(U_{w_{n}}\right)>\mu . Y_{n}=$ closure 


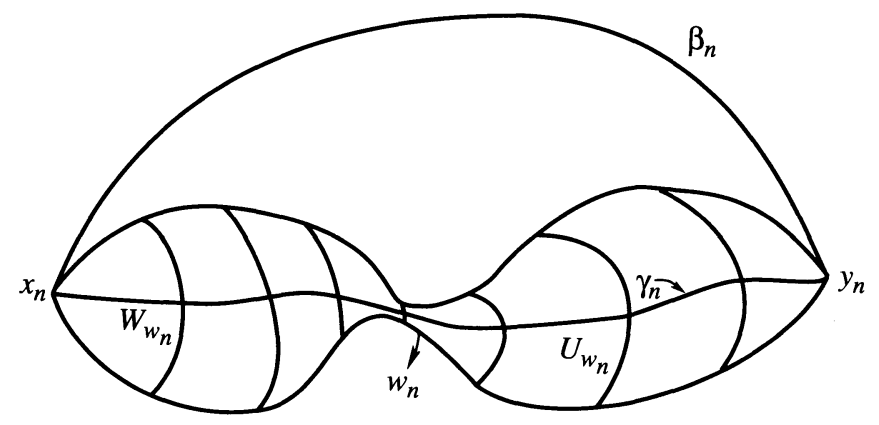

FIGURE 10. BIG QUASI-DISKS WITH SMALL QUASIGEODESICS IN THE MIDDLE.

of $W_{w_{n}}$ in $B R$ and similarly $X_{n}$ for $U_{w_{n}}$. Since they are contained in the closure of uniform quasidisks and are bounded in $\mathbf{H}^{3}$ by the quasigeodesic $w_{n}$, if $v_{d}\left(\partial Y_{n}\right) \rightarrow 0$ then $v_{d}\left(Y_{n}\right) \rightarrow 0$ and similarly for $X_{n}$. Therefore for $n$ big enough choose points $y_{n} \in \partial Y_{n}$ so that $v\left(y_{n}, w_{n}\right)>\nu$ for fixed $\nu>0$ and similarly $x_{n} \in \partial X_{n}$. In the hyperbolic structure in $\widetilde{S}_{w_{n}}$ consider the hyperbolic geodesic $\gamma_{n}^{*}$ connecting the limit points associated to $x_{n}$ and $y_{n}$, and $\gamma_{n}$ its image in $\mathbf{H}^{3}$ (see Figure 10). Let $\beta_{n}$ be the hyperbolic geodesic in $\mathbf{H}^{3}$ with the same endpoints.

Let $\alpha_{n}$ be the geodesic in $\mathbf{H}^{3}$ defined by the endpoints of $w_{n}$. Since $v_{d}\left(w_{n}\right) \rightarrow 0$ and $w_{n}$ are uniform quasigeodesics then $v_{d}\left(\alpha_{n}\right) \rightarrow 0$. As the endpoints of $\alpha_{n}$ and $\beta_{n}$ are at least $\nu$ visually apart and $v_{d}\left(\alpha_{n}\right) \rightarrow 0$, then the numbers $e_{n}=\min \left\{d_{H}\left(x, x^{\prime}\right) \mid x \in \alpha_{n}, x^{\prime} \in \beta_{n}\right\}$ satisfy $e_{n} \rightarrow+\infty$. Since $\beta_{n}$ is a bounded distance from $\gamma_{n}$, as is $\alpha_{n}$ from $w_{n}$, the minimum distance between them $\left(d_{H}\left(\gamma_{n}, w_{n}\right)\right)$ converges to $+\infty$, contradicting the fact that they intersect in $\mathbf{H}^{3}$ (because $\gamma_{n}^{*}$ and $\omega_{n}^{*}$ intersect in the intrinsic hyperbolic structure of $\widetilde{S}_{w_{n}}$ ).

Conclusion of the proof of existence. Cap off $L_{n} \times \mathbf{R}$ with the small quasi-disks given by the lemma to produce $P_{n}$. Then $P_{n}$ is topologically a 2-dimensional disk and has visual diameter converging to 0 .

It also separates $\mathbf{H}^{3}$ and the small component of $\mathbf{H}^{3}-P_{n}$ contains $\varphi_{\widetilde{T}}\left(r_{n}\right)$. Let $E_{n}$ be the closure (in $B R$ ) of this component. Since $r_{n}$ defines $p$ and the visual diameter of $E_{n}$ converges to 0 , the intersection of the $E_{n}$ defines a single point in $S_{\infty}^{2}$ which is the limit point of $\varphi_{\widetilde{T}}(r)$. This is $\bar{\varphi}_{\widetilde{T}}(p)$. q.e.d. 
We need a couple of preliminary results before addressing the continuity of $\bar{\varphi}_{\widetilde{T}}$. The region between any two asymptotic rays of the geodesic laminations is called a wedge.

Lemma 7.5. Consider a ray $r$ in $T$ which does not intersect $\Gamma^{u} \cup \Gamma^{s}$ transversally. Then $r$ is asymptotic to an escaping ray of $\Gamma^{u}$ (or $\left.\Gamma^{s}\right)$.

Proof. Assume the initial point of $r \notin \Gamma^{u} \cup \Gamma^{s}$. If $r \cap\left(\Gamma^{u} \cup \Gamma^{s}\right) \neq \varnothing$ then $r \subset\left(\Gamma^{u} \cup \Gamma^{s}\right)$, say $r \subset \Gamma^{s}$. As $r$ does not intersect $\Gamma^{s}$ transversally, $r$ is contained in an escaping of $\Gamma^{u}$.

Otherwise by Lemma 7.1 there is a subray $r^{\prime}$ contained in one of the ladders, say the positive one. Then the embedding of $r^{\prime}$ in $T$ is proper, for otherwise it would limit in a point in $T$ and again the homeomorphism would be reducible.

There is then a subray $s$ which is very near a component $S$ of $\partial_{+} N$. Project $s$ to $S$ using the $g$-flow, producing a ray $s \downarrow S$. By Proposition 5.4 this ray is almost geodesic and furthermore it does not intersect $\Gamma^{u} \downarrow S$ transversally. Since this lamination fills $S$, the description of Propositions 5.3 and 5.5 implies that $s \downarrow S$ is either asymptotic to a closed leaf or contained in a closed leaf. But the asymptotic spiralling to a closed leaf in $\Gamma^{u} \downarrow S$ corresponds to going out of the ladder and into the core, which is opposite to the direction of the ray $r$. Therefore $r$ projects to a closed leaf. By Proposition 5.5 there should be two escaping rays $r_{1}, r_{2}$ of $\Gamma^{u}$ (they may be the same), so that $r \downarrow S_{\varepsilon}$ is contained in the annulus bounded by $r_{1} \downarrow S_{\varepsilon}$ and $r_{2} \downarrow S_{\varepsilon}$. Therefore $r$ is asymptotic to $r_{1}$ and $r_{2}$.

Lemma 7.6. There is $n_{0}>0$ satisfying: Let $l$ a geodesic ray in $T$ starting in $B_{n}, n>n_{0}$. Let $l^{b}=$ component of $l \cap B$ containing the starting point of $l$, and $l^{c}$ (which might be empty) be the component of $l \cap C$ containing the endpoint of $l^{b}$. Then there are the following three possibilities:

(i) $l^{b}$ intersects $\Gamma^{u}$ transversally.

(ii) $l^{c}=\varnothing$ and $l$ is asymptotic to an escaping ray of $\Gamma^{u}$.

(iii) $l^{c}$ intersects $\Gamma^{u} \cup \Gamma^{s}$ transversally.

Proof. If $l^{c}=\varnothing$ and $l^{b}$ does not intersect $\Gamma^{u}$ transversally then $l$ does not intersect $\Gamma^{u} \cup \Gamma^{s}$ transversally and it is asymptotic to an escaping ray of $\Gamma^{u}$ by Lemma 7.5 .

Suppose the lemma is false. Then there are $l_{n}$ with starting point in $B_{n}$ with $l_{n}^{c} \neq \varnothing$ (implying $l_{n}$ not asymptotic to an escaping ray of $\Gamma^{u}$ ) and $l_{n}^{b} \cup l_{n}^{c}$ not intersecting $\Gamma^{s} \cup \Gamma^{u}$ transversally.

By Proposition 5.7, $l_{n}^{c}$ is a bounded length segment. Let $e_{n} \in \partial C$ be the endpoint of $l_{n}^{c}$ which is not contained in $l_{n}^{b}$. By taking a convergent 
subsequence we may assume $e_{n}$ converges to $e$ and the direction of $l_{n}$ at $e_{n}$ also converges. Then $l_{n}^{b} \cup l_{n}^{c}$ (finite geodesic segments) converge to a geodesic ray $l$ starting in $e$ which does not intersect $\Gamma^{u} \cup \Gamma^{s}$ transversally. By the previous lemma $l$ is asymptotic to an escaping ray of $\Gamma^{u}$ and projects to a closed leaf of $\Gamma^{u} \downarrow S$. Therefore $l$ is eventually contained in the closure of a wedge $W$. If $l \subset \Gamma^{u}$ we are done, for $l^{c}$ would have to intersect $\Gamma^{s}$ transversally. Else we can assume there is no leaf of $\Gamma^{u}$ in the wedge, for such a leaf would have to be escaping and there are finitely many of those. If $W \cap \mathscr{U}_{-} \neq \varnothing$ then $W \subset \mathscr{U}_{-}$because $\Gamma^{u}=\partial \mathscr{U}_{-}$ and $\Gamma^{u} \cap W=\varnothing$. Therefore the $g^{n}\left(\partial_{-} C\right)$ have to limit on $\partial W \subset \Gamma^{\bar{u}}$ from the interior of the wedge as well. But since the geodesic rays in $\partial W$ are asymptotic and $g^{n}\left(\partial_{-} C\right)$ is geodesic and closed this is impossible. Therefore $W \subset T-\left(\Gamma^{u} \cup \mathscr{U}_{-}\right)$, so $W \subset \Delta_{i}^{+}$for some $i$. Since $W \subset B$, it follows that $W$ is contained in a cusp. Recall that any geodesic ray in the cusp has to intersect $\Gamma^{s}$ in the opposite direction, which implies that $l^{c}$ intersects $\Gamma^{s}$ transversally, a contradiction.

Theorem 7.7. The map $\bar{\varphi}_{\widetilde{T}}: \widetilde{T}=\mathbf{H}^{2} \cup S_{\infty}^{1} \rightarrow \mathbf{H}^{3} \cup S_{\infty}^{2}$ is continuous.

Proof. Case 1. Every ray defining $p$ keeps intersecting $\Gamma^{u} \cup \Gamma^{s}$ transversally.

The $L_{n}$ described in the existence theorem form a neighborhood base of $p$ and the corresponding $E_{n}$ form a neighborhood base of $\bar{\varphi}_{\widetilde{T}}(p)$ in the limit set of $N$. Let $I_{n}$ be the component of $\mathbf{H}^{2}-L_{n}$ containing $p$. If $y$ is any point in $\mathbf{H}^{2} \cup S_{\infty}^{1}$ near enough $p$ then $y \in I_{n}$ for $n$ big and its image is contained in $E_{n}$, which has small visual diameter, therefore is near $\bar{\varphi}_{\widetilde{T}}(p)$.

Case 2. There is a ray $r$ defining $r$ defining $p$ which does not intersect $\Gamma^{u} \cup \Gamma^{s}$ transversally.

By Lemma 7.5, $r$ is asymptotic to an escaping ray so we can choose $r$ to be an escaping ray of $\Gamma^{u} \cup \Gamma^{s}$. Suppose it is contained in the positive ladder, so $r \subset \Gamma^{u}$. There might be other escaping rays of $\Gamma^{u}$ asymptotic to $r$ but there are only finitely many (see [5]). Choose $r_{1}, r_{2}$ outermost ones. If they are different there is a wedge $W$ between them. Notice no leaf of $\Gamma^{u}$ outside $W$ has limit point $p$. Since they are asymptotic the whole wedge has image converging to $\bar{\varphi}_{\widetilde{T}}(p)$. We show continuity on the outer side of $r_{1}$. Assume $r=r_{1}$ and let $z$ be its starting point, chosen to be in $B_{n}, n>n_{0}$.

We foliate a neighborhood $U$ of that side of $r$ by geodesic rays, as follows. Let $\tau$ be a transverse segment to $r$ on the side considered. If $\tau$ intersects $\Gamma^{u}$ in points other than $z$ choose a subsegment of it with an 


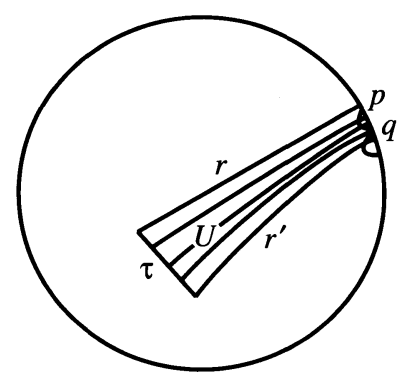

(a)

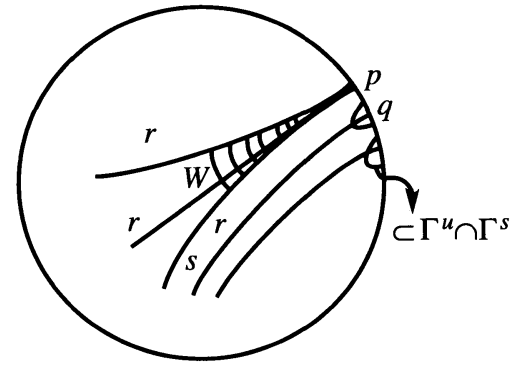

(b)

Figure 11. The LOCAL PICTURE NEAR ESCAPING RAYS.

endpoint in $\Gamma^{u}$. This always happens when $r$ is not isolated in $\Gamma^{u}$ on that side. Then let $r^{\prime}$ be a geodesic ray in $\Gamma^{u}$ starting in this endpoint. When $r$ is isolated choose $r^{\prime}$ to be a geodesic ray with ideal point close but different from $p$. Let $U$ be the set of $\mathbf{H}^{2}$ bounded by $r, r^{\prime}$, and $\tau$. Foliate $U$ with geodesic rays $s$ starting in $\tau$ so that if the starting point is in $\tau \cap \Gamma^{u}$ then the corresponding ray is contained in $\Gamma^{u}$. We do this to insure that the nearby rays do not intersect $\Gamma^{u} \cap \Gamma^{s}$ transversally near $z$ (see Figure 11(a)). For any ideal point $q$ near $p$ there is a leaf $s$ of this foliation converging to it.

The rays $s$ can only intersect $\Gamma^{u} \cup \Gamma^{s}$ transversally in leaves that are far away from $z$ in $\widetilde{T}$ (not only the intersection point is far away but so is the whole leaf). This is true for $\Gamma^{u}$ by construction and because no ray of $\Gamma^{u} \cap U$ can be asymptotic to $r$. As for $\Gamma^{s}$ note that a nearby ray $s$ stays near $r$ for a long time and can't intersect $\Gamma^{s}$ for a long time and also rays of $\Gamma^{s}$ cannot have $p$ as ideal point. The nearer $s$ is to $r$, the farther is any leaf of $\Gamma^{u} \cap \Gamma^{s}$ which intersects it transversally.

Let $\bar{\varphi}_{S}(\widetilde{S})$ be the component of $\partial_{+} \tilde{N}$ to which $x=\bar{\varphi}_{\widetilde{T}}(z)$ projects by the $g$ (or $f$ ) flow. Given $\sigma>0$ let $s_{\sigma}$ be the complement in $s$ of its starting segment of length $\sigma$. We will show that given $\xi>0$ there is $\sigma>0$ so that, for any near enough ray $s$ to $r, \bar{\varphi}_{\widetilde{T}}\left(s_{\sigma}\right)$ is $\xi$ visually near $\bar{\varphi}_{\widetilde{T}}(p)$, which implies continuity at $p$.

Lemma 7.8. Let $\xi>0$. There is $\sigma<0$ so that if the ray's is in the ladder and close enough to $r$ then $\bar{\varphi}_{\widetilde{T}}\left(s_{\sigma}\right)$ will be $\xi$ close to $\bar{\varphi}_{\widetilde{T}}(p)$.

Proof. Here we use the original $f$-flow instead of the $g$-flow because $f$ is an isometry in the ladders. The nearby arc $\bar{\varphi}_{\widetilde{T}}(s)$ will project by the original flow to the component $\bar{\varphi}_{S}(\widetilde{S})$ of $\partial \widetilde{N}$ because its starting point does. The hyperbolic thickness of the spiral in $\tilde{N}$ is bounded by $b^{\prime}>0$. 
Therefore $\bar{\varphi}_{\widetilde{T}}(r)$ and $\bar{\varphi}_{\widetilde{T}}(s)$ are moved at most a distance $b^{\prime}$ by this projection. Now choose $c>b^{\prime}$ so that any segment of $\mathbf{H}^{3}$ of length $\leq b^{\prime}$ which is farther than $c$ from $\bar{\varphi}_{\widetilde{T}}(z)$ has visual diameter $\leq \xi / 4$. Let $e>0$ so that if $x \in \widetilde{T}$ satisfies $d_{T}(x, z)>e$ then $d_{H}\left(\bar{\varphi}_{\widetilde{T}}(x), \bar{\varphi}_{\widetilde{T}}(z)\right)>2 c$. This is possible because $\bar{\varphi}_{\widetilde{T}}(\widetilde{T})$ is properly embedded in $\mathbf{H}^{3}$-otherwise there would be null homotopic closed transversals to $\mathscr{F}$.

Let $f_{s}$ be the projection of the " $S$ " ladder of $\widetilde{T}$ to $\tilde{S}$ using the map $f$. This is an isometry in the intrinsic hyperbolic metrics, so $f_{S}(r)$ and $f_{S}(s)$ are geodesics in $\tilde{S}$. Their images in $\mathbf{H}^{3}$ will be quasigeodesics. If the starting points and directions of $f_{S}(r)$ and $f_{S}(s)$ (as seen in $\tilde{S}$ ) are close enough they will be visually close in $\widetilde{S}=\mathbf{H}^{2}$ including endpoints and because $\rho\left(\pi_{1}(S)\right)$ is quasi-Fuchsian their images in $\mathbf{H}^{3}$ will be $\xi / 4$ visually close.

Consider $\bar{\varphi}_{\widetilde{T}}\left(s_{e}\right)$ and $\bar{\varphi}_{\widetilde{T}}\left(r_{e}\right)$. These rays are moved $\leq b^{\prime}$ hyperbolically to $\bar{\varphi}_{S}(\widetilde{\boldsymbol{S}})$, therefore by the above they move $\leq \xi / 4$ visually (since $\left.d_{H}\left(\bar{\varphi}_{T}\left(r_{\sigma}\right), \bar{\varphi}_{T}(z)\right)>2 c\right)$. If $s$ is close enough to $r$ then their images are $\xi / 4$ visually close in $\bar{\varphi}_{S}(\widetilde{S})$. As $\bar{\varphi}_{\widetilde{T}}(r)$ converges to $\bar{\varphi}_{\widetilde{T}}(p)$ choose a subray $r_{\sigma}$ of $r, \sigma>e$, so that $\bar{\varphi}_{\widetilde{T}}\left(r_{\sigma}\right)$ is $\xi / 4$ close to $\bar{\varphi}_{\widetilde{T}}(p)$. The total distance from $\bar{\varphi}_{\widetilde{T}}(p)$ to the corresponding subarc $\bar{\varphi}_{\widetilde{T}}\left(s_{\sigma}\right)$ of $\bar{\varphi}_{\widetilde{T}}(s)$ is less than

$\xi / 4$ from $\bar{\varphi}_{\widetilde{T}}(p)$ to $\bar{\varphi}_{\widetilde{T}}\left(r_{\sigma}\right)$,

$+\xi / 4$ from $\bar{\varphi}_{\widetilde{T}}\left(r_{\sigma}\right)$ to $\bar{\varphi}_{S}\left(f_{S}\left(r_{\sigma}\right)\right)$ along the $f$-flow,

$+\xi / 4$ from $\bar{\varphi}_{S}\left(f_{S}\left(r_{\sigma}\right)\right)$ to $\bar{\varphi}_{S}\left(f_{S}\left(s_{\sigma}\right)\right)$ in $\bar{\varphi}_{S}(\widetilde{S})$,

$+\xi / 4$ from $\bar{\varphi}_{S}\left(f_{S}\left(s_{\sigma}\right)\right)$ to $\bar{\varphi}_{\widetilde{T}}\left(s_{\sigma}\right)$ along the $f$-flow,

which sums to $\xi$. q.e.d.

If $s$ is not contained in the ladder it must intersect $\Gamma^{u} \cup \Gamma^{s}$ transversally. Take $l^{\prime}$ to be the leaf of $\Gamma^{u} \cup \Gamma^{s}$ containing the first such intersection and $s_{0}$ the subsegment of $s$ between its starting point and $l^{\prime}$. Then $d_{T}\left(l^{\prime}, z\right)$ is very big, assumed to be bigger than $\sigma$ from the previous lemma, so the visual diameter of the corresponding disc $E$ associated to $l^{\prime}$ can be made very small if $s$ is sufficiently near $r$. If $s_{0}$ is contained in the ladder the proof of the previous lemma shows that if $s$ is near enough $r$ then $\bar{\varphi}_{\widetilde{T}}\left(s_{0} \cap s_{\sigma}\right)$ is $\xi / 2$ near $\bar{\varphi}_{\widetilde{T}}(p)$. Furthermore, since $\bar{\varphi}_{\widetilde{T}}\left(s \cap l^{\prime}\right)$ is $\xi / 2$ near $\bar{\varphi}_{\widetilde{T}}(p), \bar{\varphi}_{\widetilde{T}}\left(s-s_{0}\right)$ can also be made $\xi$ near $\bar{\varphi}_{\widetilde{T}}(p)$ (if $v_{d}(E)<\xi / 2$ ).

Otherwise let $s_{0}^{\prime}$ be the connected component of $s_{0} \cap B$ intersecting $\tau$. By Lemma 7.6 $w=s_{0}-s_{0}^{\prime}$ is connected, contained in the core, and has bounded length. 
The argument now goes as follows: by the previous lemma if $s$ is near enough $r$ then $\bar{\varphi}_{\widetilde{T}}\left(s_{\sigma} \cap s_{0}^{\prime}\right)$ is visually near $\bar{\varphi}_{\widetilde{T}}(p)$. Since $w$ is far from $z, \bar{\varphi}_{\widetilde{T}}(w)$ has small visual diameter and so $\bar{\varphi}_{\widetilde{T}}(w)$ is still visually near $\bar{\varphi}_{\widetilde{T}}(p)$. As before the capped off disk associated to $l^{\prime}$ has small visual diameter which shows that the subarc $s_{\sigma}$ of $s$ also has image near $\bar{\varphi}_{\widetilde{T}}(p)$.

This completes the proof of the continuity of the extension map $\bar{\varphi}_{\widetilde{T}}: \mathbf{H}^{2}$ $\cup S_{\infty}^{1} \rightarrow \mathbf{H}^{3} \cup S_{\infty}^{2}$. q.e.d.

We have some final comments about the structure of the foliation and the limit sets of the leaves.

Even though the $g$-flow in $N$ is the appropriate tool to study the limit sets of leaves, it collapses points in $\partial N$, so obviously does not extend to a flow in the original manifold (where we modify back to $Q$ and carry back the flow). To get a good flow on $M$ put the original $f$-flow in the spiral, the new $g$-flow in the core, and a smooth transition in between. This preserves the product picture in the closure of the spiral, but still has the good dynamical properties in the core, where the invariant set $\pi\left(\left(\Gamma^{u} \times \mathbf{R}\right) \cap\left(\Gamma^{s} \times \mathbf{R}\right)\right)$ is.

Flow lines starting in $\partial_{-} \widetilde{Q}$ will either eventually hit $\partial_{+} \widetilde{Q}$ or be entirely contained in $\widetilde{Q}$, in which case they are contained in the core except for a bounded initial segment. As they are $g$-flow lines they will be flat in the $d$ semimetric and therefore quasigeodesics in $\mathbf{H}^{3}$ having a well-defined limit point in $S_{\infty}^{2}$ in the positive direction. In the same say flow lines entirely contained in $\widetilde{Q}$ are full quasigeodesics in $\mathbf{H}^{3}$ having two distinct limit points.

The foliation in $\widetilde{Q}$ is a product foliation but there is branching in $\partial \widetilde{Q}$ which accounts for the infinitely many components in $\partial \widetilde{Q}$ (see Figure 12). The limit set of $\widetilde{T}$ is $\widetilde{Q}$ is pervasive in the sense that it fills all the cracks

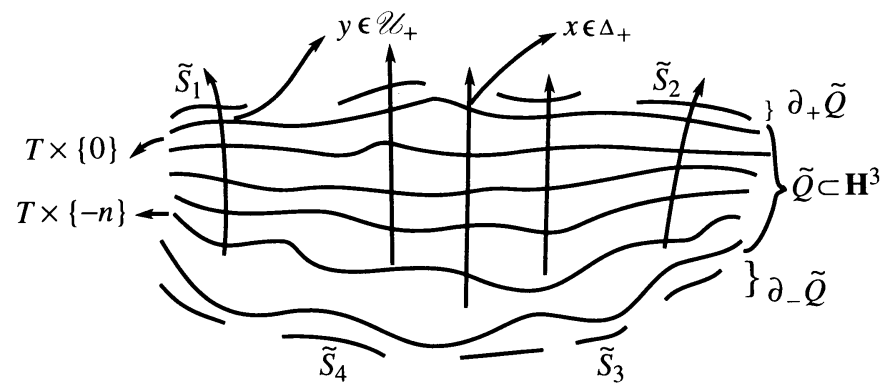

FIGURE 12. $\widetilde{Q}$ AND ITS BOUNDARY. 
in the limit set of $\widetilde{Q}$, in particular containing the limit sets of all $\widetilde{S} \subset \partial \widetilde{Q}$. For this it is essential that $\mathscr{F}$ is a fibration in $Q$.

The transverse laminations $\Gamma^{u} \times \mathbf{R}$ and $\Gamma^{s} \times \mathbf{R}$ are quasigeodesic in $\mathbf{H}^{3}$ so they appear to be quasidisks. Unfortunately they are not complete: a wall $l \times \mathbf{R}, l \in \Gamma^{s}$, is complete in the positive direction but not in the negative one. In any way they product barriers in $\widetilde{Q}$ and the capped off walls separate $\mathbf{H}^{3}$. It is not clear priori whether the capped off walls are quasigeodesic in $\mathbf{H}^{3}$ but in any case most of them will have small visual diameter.

The walls show that while the leaf $\widetilde{T}$ is not quasigeodesic - there are geodesics in $\widetilde{T}$ with endpoints identified - the limit behavior of $\widetilde{T}$ is still good locally.

\section{Acknowledgments}

This paper is based on the author's Ph.D. thesis. The author wants to thank Bill Thurston for teaching him about hyperbolic 3-manifolds and foliations, and for his guidance during graduate school; Francis Bonahon for going through the details of this paper and for innumerable suggestions and corrections; and finally Michael Handel and Lee Mosher for stimulating discussions about end periodic maps and flows on 3-manifolds.

\section{References}

[1] A. F. Beardon, The geometry of discrete groups, Graduate Texts in Math., No. 91, Springer, Berlin, 1983.

[2] J. Cannon \& W. Thurston, Group invariant peano curves, preprint.

[3] J. Cantwell \& L. Conlon, Poincaré-Bendixson theory for leaves of codimension one, Trans. Amer. Math. Soc. 269 (1981) 181-209.

[4] S. Fenley, Depth one foliations in hyperbolic 3-manifolds, Thesis, Princeton University, Princeton, NJ, 1989.

[5] __ On end periodic homeomorphisms and 3-manifolds, in preparation.

[6] D. Gabai, Foliations and the topology of 3-manifolds, J. Differential Geometry 18 (1983) 445-503.

[7] __ Foliations and genera of links, Topology 23 (1984) 381-394.

[8] S. Goodman, Closed leaves in foliations of codimension one, Comment. Math. Helv. 50 (1975) 383-388.

[9] A. Haefliger, Varietés Feilleteès, Ann. Scuola Norm. Sup. Pisa (3) 16 (1962) 367-397.

[10] M. Handel \& R. Miller, End periodic homeomorphisms, in preparation.

[11] J. Hempel, 3-manifolds, Annals of Math. Studies, No. 86, Princeton University Press, Princeton, NJ, 1976.

[12] W. Jaco. Lectures on 3-manifold topology, CMBS Regional Conf. Ser. in Math., Vol. 43, Conf. Board Math. Sci., Washington, D.C., 1980.

[13] W. Lickorish, A foliation for 3-manifolds, Ann. of Math. (2) 82 (1965) 414-420. 
[14] J. Morgan, On Thurston's uniformization theorem for 3 dimensional manifolds, The Smith Conjecture, J. Morgan \& H. Bass, eds., Academic Press, New York, 1984, 37-125.

[15] S. P. Novikov, Topology of foliations, Trans. Moscow Math. Soc. 14 (1963) 268-305.

[16] G. Reeb, Sur certaines propriétés topologiques des variétés feilletées, Actualités Sci. Indust., No. 1182, Hermann, Paris, 1982.

[17] W. Thurston, The geometry and topology of 3-manifolds, Princeton University Lecture Notes, Princeton, NJ, 1982.

[18] _ 3-dimensional manifolds, Kleinian groups and hyperbolic geometry, Bull. Amer. Math. Soc. (N.S.) (1982) 357-381.

[19] F. Waldhausen, On irreducible 3-manifolds which are sufficiently large, Ann. of Math. (2) 87 (1968) 56-88.

WASHINGTON UNIVERSITY 
